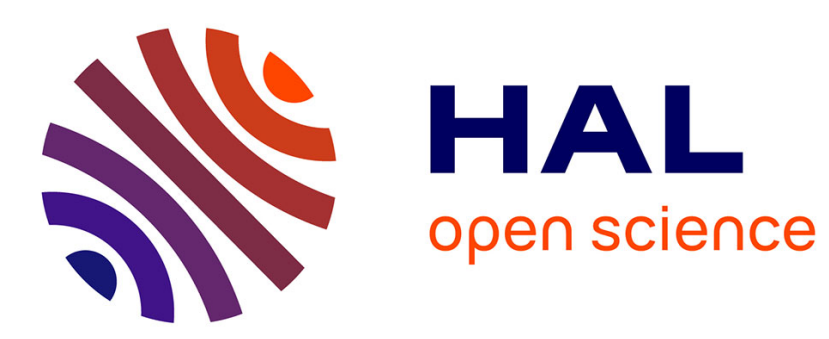

\title{
A new deviational Asymptotic Preserving Monte Carlo method for the homogeneous Boltzmann equation
} Anaïs Crestetto, Nicolas Crouseilles, Giacomo Dimarco, Mohammed Lemou

\section{To cite this version:}

Anaïs Crestetto, Nicolas Crouseilles, Giacomo Dimarco, Mohammed Lemou. A new deviational Asymptotic Preserving Monte Carlo method for the homogeneous Boltzmann equation. Communications in Mathematical Sciences, 2020, 18 (8), pp.2305-2339. 10.4310/CMS.2020.v18.n8.a10 . hal02413232

\section{HAL Id: hal-02413232 \\ https://hal.inria.fr/hal-02413232}

Submitted on 16 Dec 2019

HAL is a multi-disciplinary open access archive for the deposit and dissemination of scientific research documents, whether they are published or not. The documents may come from teaching and research institutions in France or abroad, or from public or private research centers.
L'archive ouverte pluridisciplinaire HAL, est destinée au dépôt et à la diffusion de documents scientifiques de niveau recherche, publiés ou non, émanant des établissements d'enseignement et de recherche français ou étrangers, des laboratoires publics ou privés. 


\title{
A new deviational Asymptotic Preserving Monte Carlo method for the homogeneous Boltzmann equation
}

\author{
Anaïs Crestetto* Nicolas Crouseilles ${ }^{\dagger} \quad$ Giacomo Dimarco ${ }^{\ddagger} \quad$ Mohammed Lemou $^{\S}$
}

December 16, 2019

\begin{abstract}
In this work, we introduce a new Monte Carlo method for solving the Boltzmann model of rarefied gas dynamics. The method works by reformulating the original problem through a micro-macro decomposition and successively in solving a suitable equation for the perturbation from the local thermodynamic equilibrium. This equation is then discretized by using unconditionally stable exponential schemes in time which project the solution over the corresponding equilibrium state when the time step is sent to infinity. The Monte Carlo method is designed on this time integration method and it only describes the perturbation from the final state. In this way, the number of samples diminishes during the time evolution of the solution and when the final equilibrium state is reached, the number of statistical samples becomes automatically zero. The resulting method is computationally less expensive as the solution approaches the equilibrium state as opposite to standard methods for kinetic equations which computational cost increases with the number of interactions. At the same time, the statistical error decreases as the system approaches the equilibrium state. In a last part, we show the behaviors of this new approach in comparison with standard Monte Carlo techniques and in comparison with spectral methods on different prototype problems.
\end{abstract}

Mathematics Subject Classification: 76P05, 82C80, 82D05, 65C05, 35B25

Keywords: Boltzmann equation; Monte Carlo methods; asymptotic preserving schemes; micro-macro decomposition.

\section{Introduction}

In this work, we deal with the development of efficient Monte Carlo (MC) methods for the Boltzmann equation of gas dynamics. It is well known that for many different engineering applications, the correct description of the flows cannot be achieved by using standard fluid

\footnotetext{
${ }^{1}$ Laboratoire de Mathématiques Jean Leray, CNRS UMR 6629, Université de Nantes, France. E-mail: anais . crestetto@univ-nantes.fr

${ }^{2}$ Univ Rennes, Inria Rennes \& Institut de Recherche Mathématiques de Rennes, CNRS UMR 6625 Rennes \& ENS Rennes, France. E-mail: nicolas.crouseilles@inria.fr

${ }^{3}$ Department of Mathematics and Computer Science. University of Ferrara \& Center for Modeling, Computing and Statistic Italy. E-mail: giacomo.dimarco@unife.it

${ }^{4}$ Univ Rennes, CNRS \& Institut de Recherche Mathématiques de Rennes, CNRS UMR 6625 \& Inria Rennes \& ENS Rennes, France. E-mail:. E-mail: mohammed.lemou@univ-rennes1.fr
} 
models such as compressible Euler or Navier-Stokes equations $[4,16]$. For this reason, one often relies on kinetic equations which offer a wider range of possibilities in term of modeling physical problems of different natures. This approach attracted recently even more interest thanks to modern applications which lie outside the standard rarefied gases and/or plasmas simulations such as socio-economic models, traffic and crowd dynamics, description of biological phenomena etc. [54]. In these situations kinetic equations are becoming a leading modeling approach.

It is a matter of fact that the kinetic description is a much richer way to describe physical phenomena than the hydrodynamic one. However, the price to pay relies in the larger complexity of the models. In fact, from the computational point of view, kinetic models and in particular the Boltzmann one represents a real challenge. This is due to the larger dimensional space in which kinetic equations live (six dimensions compared to the standard three dimensions in which fluid equations live) and to the nature of the Boltzmann collisional operator characterized by a five fold integral in velocity space $[16,29]$ which has to be solved locally in space. In addition, the phase space is unbounded and the physical properties which are described by the collision operator turns to be very important since they are used to derive the macroscopic state of the system. For this reason, numerical methods should, in principle, be able to reproduce the continuous behavior of the system in terms of physical conservations.

Consequently, the numerical techniques are often designed in such a way that the computational cost is as low as possible. In this context, Monte Carlo methods have been the dominant approach to deal with rarefied gases starting from last century, see $[29,16,10]$ for a review on the subject. The most known examples belonging to this class are the Direct Simulation Monte Carlo methods (DSMC) $[1,4,10,50,52,53]$. The common feature of these approaches is to evolve a finite set of particles representing the distribution function (which defines the state of the system at each instant of time) according to their velocity and to perform collisions between them according to the law described by the Boltzmann operator. The role of the collision operator being to change the velocity of the particles. Monte Carlo methods are indeed very interesting techniques since it is relatively easy to incorporate additional physical features in the schemes at the contrary to deterministic methods and they do not need artificial boundaries in velocity space. In addition, they are typically very efficient especially for stationary problems since one can easily use time averaging techniques to reduce the statistical error. However, they are poorly accurate when used to solve unsteady problems. This is particularly true for low Mach number regimes: in these cases it is very difficult to capture the correct solution due to the very low average speed of the gas $[4,16]$. Several authors tried to overcome the issue of the low convergence rate and of the numerical noise of Monte Carlo schemes in the recent past. We quote in particular the review papers $[10,55]$ for an overview on efficient and low variance Monte Carlo methods. Some recent papers which make use of these techniques have been derived in the context of kinetic equations $[21,23,24,25,12,35,36,59,42,60,17,18,7,8,15,14,13,58,19,12,31$, 48]. In particular, we recall the recent article [60] which shares similarities with the work here presented even if its focus was more on Coulomb interactions arising in plasma physics and not on rarefied gases.

Frequently, when the kinetic model is used for describing realistic problems, it also turns that different time and space scales enter into the game. For instance, a famous problem is the one related to the fluid limit, i.e. the cases in which the kinetic and the macroscopic model coincide [16]. This results in situations in which fast scales which characterize collisions cohabit with slow ones, typically the ones describing the average motion of the gas. In these cases, from 
the numerical analysis point of view the problem becomes stiff, while regarding the Monte Carlo interpretation of the solution, this translates in a number of collisions which goes to infinity. It is then very important to find numerical methods which avoid the bottleneck caused by a growing number of collisions or equivalently by the stiffness of the equations [29]. A well-known class of schemes which is designed expressly for treating multiscale problems is the Asymptotic Preserving (AP) one [3, 20, 28, 37, 43, 44, 27, 30, 26, 56, 33, 38, 40, 41]. Their main interest is that time steps are not constrained to the fast scale dynamics and that they automatically degenerate to consistent discretizations of the limiting models when the parameters which characterize the microscopic behaviors go to zero. For the above reason, they are very interesting in the context of kinetic equation, even if there do not exist many cases in which these methods have been derived specifically in synergy with Monte Carlo approaches [56, 26].

In this paper, we propose a new Asymptotic Preserving Monte Carlo method which solves the Boltzmann equation of gas dynamics. It is specifically designed to address the complexity of the underlying kinetic equation, to reduce the numerical noise of classical MC methods and to overcome the stiffness of the equation close to the fluid limit. The scheme proposed here is inspired by some recent papers on the same subject $[24,25,21,17,60]$ while improving the results obtained. In details, we focus on the space homogeneous problem and we design the Monte Carlo method by rewriting the equation in term of the time evolution of the perturbation from equilibrium. Then, we use exponential Runge-Kutta methods to discretize the resulting equation. Particles are successively used to describe only the perturbation from the equilibrium and a Monte Carlo interpretation of the resulting equation is furnished. One of the major problem when this kind of MC approach is used is that the total number of particles increases with time due to collisions with particles sampled from the equilibrium state [59, 60, 42]. Here, we solve this problem by using a subset of samples to estimate the distribution function shape through kernel density reconstruction techniques [6] and then we use this estimate as a probability for discarding or keeping particles through an acceptance-rejection algorithm [53]. This approach permits to eliminate samples which give redundant information at a cost proportional to the number of samples which are present at a fixed time of the simulation in the domain. In this way, the method enjoys both the unconditional stability property and the complexity reduction one as the solution approaches the thermodynamic equilibrium. In fact, the particles are used to describe only the perturbation which goes to zero exponentially fast and thus disappear exponentially fastly. Thus, the statistical error due to the MC method decreases as the number of interactions increases, realizing a variance reduction method which effectiveness depends on the regime studied. Far from equilibrium the same variance of classical MC methods is obtained, while close to equilibrium the variance is lower than that of a classical MC. The approach presented here can then be incorporated in a solver for the spatially non homogeneous case by coupling it with deterministic methods for the equilibrium part of the solution. We do not discuss this issue here and we refer to a future work to extend the present method to the non homogeneous case.

The remainder of the paper is organized as follows. In the next Section we recall some basics about the Boltzmann equation together with some notions of splitting methods. Classical Monte Carlo approaches are discussed in Section 3. A micro-macro reformulation and an asymptotic preserving exponential time integrator are discussed in Section 4. In Section 5, we then give the details of the new Monte Carlo method. Numerical results are presented in Section 6, where we compare the classical MC method with the new AP one and with spectral methods 
for the Boltzmann operator. Section 7 is used to draw some conclusions and suggest future developments. In Appendix are reported some details about the spectral methods used for the comparisons and the details of an alternative asymptotic preserving Monte Carlo algorithm.

\section{The Boltzmann equation and the splitting approach}

In this Section we briefly recall the Boltzmann equation [16] and some basics on splitting in time methodologies [29].

In kinetic theory, the non-negative function $f(x, v, t)$, called distribution function, characterizes the state of the system and it gives the probability for a particle to have velocity $v \in \mathbb{R}^{d_{v}}$ in position $x \in \mathbb{R}^{d_{x}}$ at time $t \in \mathbb{R}^{+}$, where $d_{x}$ is the physical dimension and $d_{v}$ the dimension of the velocity space. Despite its stochastic nature, the probability distribution evolution is given by a deterministic partial differential equation

$$
\frac{\partial f}{\partial t}+v \cdot \nabla_{x} f=Q(f, f)
$$

The operator $Q(f, f)$, on the right hand side in equation (1), describes the effects of particle interactions and its form depends on the details of the microscopic dynamics chosen. Here, we focus our attention on the Boltzmann operator of gas dynamics. It reads

$$
Q(f, f)(v)=\int_{\mathbb{R}^{d_{v}}} \int_{\mathbb{S}^{d_{v}-1}} B\left(\left|v-v_{*}\right|, \omega\right)\left(f\left(v^{\prime}\right) f\left(v_{*}^{\prime}\right)-f(v) f\left(v_{*}\right)\right) d v_{*} d \omega,
$$

where, in the above equation, we have highlighted its bilinear nature and where $\omega$ is the vector of the unitary sphere $\mathbb{S}^{d_{v}-1} \subset \mathbb{R}^{d_{v}}$ defined by

$$
\omega=\frac{v^{\prime}-v}{\left|v^{\prime}-v\right|}
$$

The pre-collisional velocities $\left(v^{\prime}, v_{*}^{\prime}\right)$ in $(2)$ are related with the post-collisional ones $\left(v, v_{*}\right)$ by

$$
v^{\prime}=v+\left\langle v-v_{*}, \omega\right\rangle \omega, \quad v_{*}^{\prime}=v_{*}-\left\langle v-v_{*}, \omega\right\rangle \omega,
$$

where $\langle\cdot, \cdot\rangle$ denotes the scalar product in $\mathbb{R}^{d_{v}}$. The kernel $B$ characterizes the details of the binary interactions, it has the general form

$$
B\left(\left|v-v_{*}\right|, \omega\right)=B\left(\left|v-v_{*}\right|, \cos \theta\right)=\left|v-v_{*}\right| \sigma\left(\left|v-v_{*}\right|, \cos \theta\right)
$$

where $\sigma$ is the scattering cross-section and $\theta$ the scattering angle. A general model for interactions gives

$$
\left|v-v_{*}\right| \sigma\left(\left|v-v_{*}\right|, \cos \theta\right)=b_{\alpha}(\cos \theta)\left|v-v_{*}\right|^{\alpha},
$$

with $\alpha=(k-5) /(k-1)$ and in the situation $k=5$, the model is said the Maxwell pseudomolecules model. In the following, we will consider the Hard Sphere model [4] for which $\sigma(\mid v-$ $\left.v_{*} \mid, \cos \theta\right)=C_{\alpha}\left|v-v_{*}\right|^{\alpha-1}$, with $C_{\alpha}$ a given positive constant, in addition we fix $k=5$. The extension of the presented method to more general cases is postponed to future investigations. The type of interactions considered provides the family of solutions for the velocities

$$
\begin{array}{r}
v+v_{*}=v^{\prime}+v_{*}^{\prime}, \\
|v|^{2}+\left|v_{*}\right|^{2}=\left|v^{\prime}\right|^{2}+\left|v_{*}^{\prime}\right|^{2},
\end{array}
$$


which expresses at the kinetic level the conservation of momentum and energy of the system. These conservation properties together with the conservation of the number of particles (equivalently of the mass) can be conveniently written as

$$
\int_{\mathbb{R}^{d v}} Q(f, f)(v) \phi(v) d v=0
$$

where $\phi(v)=\left(1, v, \frac{1}{2}|v|^{2}\right)$ are commonly called the collision invariants. It is possible to show that the functions belonging to the kernel of the collision operator, as it has been defined, satisfy

$$
Q(f, f)=0 \quad \text { iff } \quad f=M[f]
$$

where the Maxwellian distributions $M[f]=M[f](x, v, t)$ is defined as the normal distribution which moments $U_{M}(x, t)=\int_{\mathbb{R}^{d_{v}}} M(x, v, t) \phi(v) d v$ match those of the distribution function $f$

$$
\int_{\mathbb{R}^{d v}} M[f](x, v, t) \phi(v) d v=U_{M}(x, t)=U(x, t)=\int_{\mathbb{R}^{d_{v}}} f(x, v, t) \phi(v) d v \in \mathbb{R}^{2+d_{v}} .
$$

This distribution reads

$$
M[f](x, v, t)=\frac{\rho(x, t)}{(2 \pi T(x, t))^{d_{v} / 2}} e^{-\frac{|v-u(x, t)|^{2}}{2 T(x, t)}},
$$

where $\rho, u$ and $T$ are respectively the density, the mean velocity and the temperature of $f$ and are linked to $U(x, t)=(\rho, \rho u, E)(x, t)$ using the relation $d_{v} \rho T=2 E-\rho|u|^{2}$.

We discuss now the operator splitting approach. A large part of the literature on numerical methods for kinetic equations is based on a splitting in time between free particle transport and collisions $[4,29]$. The extension of the method here described to the full non homogeneous case can then be derived following this theory. The starting point is given by an operator splitting of (1) in a time interval $[0, \Delta t]$ between relaxation

$$
\partial_{t} f=Q(f, f)
$$

and free transport

$$
\partial_{t} f+v \cdot \nabla_{x} f=0 .
$$

This situation is typical of Monte Carlo methods and of several other numerical methods used in realistic simulations. In the case of MC methods, the second part consists in a simple shift of the particles at speed $v$ and it does not require any spatial mesh to be set into practice. The above splitting, usually referred to as Lie-Trotter splitting, is limited to first order in time. Higher order splitting formulas can be derived in different ways (see [34]). For example by denoting $\mathcal{T}_{\Delta t}(f)$ and $\mathcal{C}_{\Delta t}(f)$ the transport and collision part of the kinetic equation in a time interval $[0, \Delta t]$ starting from the initial data $f_{0}$, the well-known second order Strang splitting [57] can be written as

$$
\mathcal{C}_{\Delta t / 2}\left(\mathcal{T}_{\Delta t}\left(\mathcal{C}_{\Delta t / 2}\left(f_{0}\right)\right)\right)
$$

Splitting schemes of order higher than two with real coefficients necessarily involve negative weights [34]. This is the source of important drawbacks which make very difficult their use in Monte Carlo schemes. 


\section{Monte Carlo methods}

We introduce here some classical Monte Carlo approaches which can be used for computing an approximate solution of the Boltzmann equation [53]. Namely, we focus on the methods of Nanbu [50] and of Nanbu-Babovsky [1]. From now on, we restrict ourselves to the simplified case $f=f(v, t)$ since we do not consider any spatial dependence. Let us observe that the Boltzmann operator can be written as the sum of a gain and a loss term:

$$
Q(f, f)=Q^{+}(f, f)-Q^{-}(f, f) .
$$

The loss term counts the collisions in which a given particle $v$ encounters a particle with velocity $v_{*}$. This type of interaction leads to a change in velocity and consequently in a loss of the particle with the velocity $v$. On the other hand, the gain term counts the collisions in which a particle with speed $v^{\prime}$ meets a so-called test particle with velocity $v_{*}^{\prime}$ and the interaction gives rise to a particle with speed $v$. Having said so, we restrict our analysis to situations in which the homogeneous Boltzmann equation can be written as

$$
\frac{\partial f}{\partial t}=P(f, f)-f \int_{\mathbb{R}^{d_{v}}} \int_{\mathbb{S}^{d_{v}-1}} b(\omega) f\left(v_{*}\right) d v_{*} d \omega
$$

where $b$ is defined through (5) and

$$
P(f, f)(v)=\int_{\mathbb{R}^{d v}} \int_{\mathbb{S}^{d v}-1} b(\omega) f\left(v^{\prime}\right) f\left(v_{*}^{\prime}\right) d v_{*} d \omega
$$

i.e. the Maxwellian molecules case. Let now introduce the following first order in time Euler discretization using $f^{n}(v) \approx f(v, n \Delta t), \forall n \in \mathbb{N}$ (with $\Delta t>0$ the time step)

$$
f^{n+1}=\left(1-\Delta t \int_{\mathbb{R}^{d v}} \int_{\mathbb{S}^{d v}-1} b(\omega) f^{n}\left(v_{*}\right) d v_{*} d \omega\right) f^{n}+\Delta t P\left(f^{n}, f^{n}\right) .
$$

In a Monte Carlo method, the distribution function $f(v, t)$ is approximated by a finite set of $N$ particles

$$
f(v, t) \approx m \sum_{k=1}^{N} \delta_{v_{k}(t)}(v)
$$

with $v_{k}(t) \in \mathbb{R}^{d_{v}}$ the particles velocities and $m>0$ a constant weight, computed from the following expression

$$
m=\frac{1}{N} \int_{\mathbb{R}^{d_{v}}} f(v, t=0) d v .
$$

This states that the product $N m$ has to (statistically) match the density of the particles. In this setting, we say that at a given time $t$, the particle approximation (17) converges to $f(v, t)$ if

$$
\lim _{N \rightarrow \infty} \sum_{k=1}^{N} m \varphi\left(v_{k}(t)\right)=\int_{\mathbb{R}^{d v}} f(v, t) \varphi(v) d v, \quad \text { for all } \varphi \in C^{b}\left(\mathbb{R}^{d_{v}}\right)
$$

i.e. if the discrete measure $m \sum_{k=1}^{N} \delta_{v_{k}(t)}(v)$ converges in the weak* topology to $f(v, t) d v$. 
The goal of the sequel is to make the link between (16) and the time strategy to advance the particles velocities through the Monte Carlo method. Now, multiplying equation (16) by a test function $\varphi(v) \in C^{b}\left(\mathbb{R}^{d_{v}}\right)$ and integrating in velocity, one gets

$$
\begin{aligned}
\int_{\mathbb{R}^{d_{v}}} \varphi(v) f^{n+1}(v) d v= & \int_{\mathbb{R}^{d_{v}}}\left(1-\Delta t \int_{\mathbb{R}^{d_{v}}} \int_{\mathbb{S}^{d_{v}-1}} b(\omega) f^{n}\left(v_{*}\right) d v_{*} d \omega\right) f^{n}(v) \varphi(v) d v \\
& +\Delta t \int_{\mathbb{R}^{d_{v}}} P\left(f^{n}, f^{n}\right) \varphi(v) d v \\
= & \int_{\mathbb{R}^{d_{v}}} \int_{\mathbb{R}^{d_{v}}} \varphi(v)\left(1-\Delta t \int_{\mathbb{S}^{d_{v}-1}} b(\omega) d \omega\right) f^{n}\left(v_{*}\right) f^{n}(v) d v_{*} d v \\
& +\int_{\mathbb{R}^{d_{v}}} \int_{\mathbb{R}^{d_{v}}} \Delta t \int_{\mathbb{S}^{d_{v}-1}} b(\omega) \varphi\left(v^{\prime}\right) d \omega f^{n}(v) f^{n}\left(v_{*}\right) d v_{*} d v \\
= & \int_{\mathbb{R}^{d_{v}}} \int_{\mathbb{R}^{d_{v}}} \int_{\mathbb{S}^{d_{v}-1}}\left(K_{v, v_{*}, \omega} \varphi\right) f^{n}(v) f^{n}\left(v_{*}\right) \chi_{\mathbb{S}^{d_{v}-1}}(\omega) d v d v_{*} d \omega
\end{aligned}
$$

where $\chi_{\mathbb{S}^{d_{v}-1}}$ is the characteristic function of $\mathbb{S}^{d_{v}-1}$ and where we introduced $K_{v, v_{*}, \omega} \varphi$ defined by the following expression

$$
K_{v, v_{*}, \omega} \varphi=(1-\Delta t b(\omega)) \varphi(v)+\Delta t b(\omega) \varphi\left(v^{\prime}\right),
$$

with $v^{\prime}=v+\left\langle v-v_{*}, \omega\right\rangle \omega$. In the above relations, we used the classical properties (involution, preservation of energy and momentum) of the transformation $\left(v, v_{*}\right) \mapsto\left(v^{\prime}, v_{*}^{\prime}\right)$ defined by (3). Denoting by $T\left(v, v_{*}, \omega\right)$ the transformation that gives rise to a particle with velocity $v^{\prime}$ from a collision of a particle $v$ with a particle with velocity $v_{*}$ (see $(3)$ ), one now can derive a particle approximation of $f^{n+1}(v)$. Indeed, from Theorem 1 of [51], if $\frac{m}{N} \sum_{k=1}^{N} \delta_{\left(v_{k}^{n}, v_{*, k}^{n}, \omega_{k}^{n}\right)}$ converges weakly towards $f^{n}(v) f^{n}\left(v_{*}\right) \chi_{\mathbb{S}^{d} v^{-1}}(\omega) d v d v_{*} d \omega$, then (let recall that we are considering the Hard Sphere case so that $b(\omega)=C \in \mathbb{R})$

$$
\begin{aligned}
\int_{\mathbb{R}^{d_{v}}} \varphi(v) f^{n+1}(v) d v & \approx \frac{m}{N} \sum_{k=1}^{N} \int_{\mathbb{R}^{d_{v}}} \int_{\mathbb{R}^{d_{v}}} \int_{\mathbb{S}^{d^{d}-1}}\left(K_{v, v_{*}, \omega} \varphi\right) \delta_{\left(v_{k}^{n}, v_{*, k}^{n}, \omega_{k}^{n}\right)} d v d v_{*} d \omega \\
& \approx \frac{m}{N} \sum_{k=1}^{N}\left[(1-\Delta t C) \varphi\left(v_{k}^{n}\right)+\Delta t C \varphi\left(T\left(v_{k}^{n}, v_{*, k}^{n}, \omega_{k}^{n}\right)\right)\right] .
\end{aligned}
$$

Finally, to build up an approximation of the measure product $f^{n}(v) f^{n}\left(v_{*}\right) d v d v_{*}$, it is sufficient to choose uniformly pairs of particles out of the set $\left\{v_{k}^{n}\right\}_{k=1, \ldots, N}$ (see $[51,1]$ ). In the following we recall two classical methods based on the above computations. First we define

$$
\mu=\int_{\mathbb{R}^{d v}} \int_{\mathbb{S}^{d v-1}} b(\omega) f^{n}(v) d \omega d v .
$$

Then, rewriting (16) as

$$
f^{n+1}=(1-\mu \Delta t) f^{n}+\mu \Delta t \frac{P\left(f^{n}, f^{n}\right)}{\mu},
$$

we have the following probabilistic interpretation:

From a given particle with velocity $v_{k}^{n}$ 
- with probability $(1-\mu \Delta t)$, the particle does not collide, i.e. $v_{k}^{n+1}=v_{k}^{n}$.

- with probability $\mu \Delta t$, the particle collides according to the collision law defined by the normalized operator $P\left(f^{n}, f^{n}\right) / \mu$ : i.e. $v_{k}^{n+1}=v_{k}^{n}-\left\langle v_{k}^{n}-v_{j}^{n}, \omega_{k}^{n}\right\rangle \omega_{k}^{n}$, where $v_{j}^{n}$ is chosen randomly.

This corresponds to the Nanbu algorithm [50] which can be written in this case as

Algorithm 3.1. Nanbu for Maxwell molecules (see [53]).

- Compute the initial velocities of the particles: $\left\{v_{1}^{0}, . ., v_{N}^{0}\right\}$

- from $n=1$ to $n=n_{\text {fin }}$

- for particles from $k=1$ to $k=N$

* with probability $(1-\mu \Delta t)$ set $v_{k}^{n+1}=v_{k}^{n}$

* with probability $\mu \Delta t$ :

a) select a random particle $j$ (whose velocity is $v_{j}^{n}$ )

b) compute $v_{k}^{\prime}$ by performing a collision between particle $k$ and particle $j$

c) assign $v_{k}^{n+1}=v_{k}^{\prime}:=v_{k}^{n}-\left\langle v_{k}^{n}-v_{j}^{n}, \omega_{k}^{n}\right\rangle \omega_{k}^{n}$.

- end loop over the particles

- end loop over time

In the above algorithm $n_{\text {fin }}$ corresponds to the final time iteration $n_{\text {fin }} \Delta t=T_{\text {fin }}$, the collision law is defined by (3) and the time step $\Delta t<1 / \mu$ for the probabilistic interpretation to hold true. Let observe that, the Nanbu method is not exactly conservative. In fact, both energy and average velocity are conserved only in the mean but not at each collision. A conservative version of the Nanbu scheme has been introduced by Babovsky [1]. We recall it briefly

Algorithm 3.2. Nanbu-Babovsky for Maxwell molecules (see [53]).

- Compute the initial velocities of the particles: $\left\{v_{1}^{0}, . ., v_{N}^{0}\right\}$

- from $n=1$ to $n=n_{\text {fin }}$

- set $N_{c}=\operatorname{Iround}(\mu N \Delta t / 2)$ and select $N_{c}$ pairs $(i, j)$ uniformly among all possible pairs. For those pairs

a) compute $v_{i}^{\prime}$ and $v_{j}^{\prime}$ according to the collision law (3)

b) assign $v_{i}^{n+1}=v_{i}^{\prime}$ and $v_{j}^{n+1}=v_{j}^{\prime}$

- set $v_{k}^{n+1}=v_{k}^{n}$ for all other particles which have not collided.

- end loop over time

The operator $\operatorname{Iround}(x)$ in the above description simply indicates a stochastic rounding of the real number $x$ towards the closest integer

$$
\operatorname{Iround}(x)= \begin{cases}\lfloor x\rfloor+1, & \text { with probability } x-\lfloor x\rfloor \\ \lfloor x\rfloor, & \text { with probability } 1-x+\lfloor x\rfloor\end{cases}
$$

Finally, the collision angle $\omega$ which is used for computing the post-collisional velocities in the two MC algorithms is randomly chosen uniformly in the unit sphere. 


\section{Asymptotic Preserving methods}

The Monte Carlo methods described in the previous Section deserve some remarks. First, the typical error estimate depends upon the number of particles chosen as $\mathcal{O}\left(N^{-1 / 2}\right)$ and the variance of the distribution function $f(v, t)$, thus convergence is as well known quite slow. In addition, we have a requirement on the time step that should be smaller than a given constant $\mu$ to ensure positivity, stability and a MC interpretation of the time integration formula. This requirement becomes typically very restrictive when dealing with non homogeneous problems close to the fluid limit.

For the above reasons, we start now to discuss possible remedies which can improve the MC approach. We start by deriving a suitable time integration scheme that deals with the problem of the stiffness close to the fluid limit. In order to highlight this stiffness, even if we are working in the space homogeneous case and thus we have only one time scale, we rescale the Boltzmann equation by $\tilde{t}=t / \varepsilon$ in such a way that the Knudsen number $\varepsilon$ appears at the denominator in front of the collision operator $Q(f, f)$. We also recall $\tilde{t}$ with $t$ for simplicity to get

$$
\partial_{t} f=\frac{1}{\varepsilon} Q(f, f)
$$

We give now a definition of Asymptotic Preserving scheme and some related properties in this context and we recall a scheme (proposed in [26]) which enjoys the AP property.

Definition 4.1. A consistent time discretization method for (25) of stepsize $\Delta t$ is asymptotic preserving (AP) if, independently of the initial data and of the stepsize $\Delta t$, in the limit $\varepsilon \rightarrow 0$, it projects the distribution function over the equilibrium state: $f(v, t)=M[f](v, t)$.

Let observe that the above definition coincides with the L-stability definition when one considers the so called test problem of ODEs: $y^{\prime}=-\lambda y$. Let also observe that, in the case of a non homogeneous problem, fulfilling the above request is sufficient to have an AP method in the case in which splitting methods are employed [26]. We also introduce the notion of entropic stability, namely schemes that preserve the entropy inequality

$$
\frac{d}{d t} \int_{\mathbb{R}^{d_{v}}} f(v, t) \log f(v, t) d v=\frac{1}{\varepsilon} \int_{\mathbb{R}^{d_{v}}} Q(f, f)(v, t) \log f(v, t) d v \leq 0,
$$

which is known to be satisfied by the Boltzmann equation at the continuous level. This definition reads as follows.

Definition 4.2. A time discretization method for (10) is called unconditionally entropic if $H\left(f^{n+1}\right) \leq H\left(f^{n}\right)$, where $H(f)=\int_{\mathbb{R}^{3}} f(v, t) \log f(v, t) d v$, independently of the step size $\Delta t$.

Since we aim at developing unconditionally stable schemes, the most natural choice would be to use implicit solvers applied to (25). Unfortunately, the use of implicit schemes in combination with Monte Carlo methods is a difficult task to be set into practice. The alternative we propose consists in rewriting once again the homogeneous Boltzmann equation (25) separating the gain from the loss term where, in addition, we stick to the Maxwellian molecules case. We then have

$$
\partial_{t} f=\frac{1}{\varepsilon}(P(f, f)-\mu f),
$$


where $\mu$ is defined in $(22)$ and $P(f, f)$ in (15). Using (7), we have

$$
\frac{1}{\mu} \int_{\mathbb{R}^{d v}} \phi(v) P(f, f)(v, t) d v=\int_{\mathbb{R}^{d v}} \phi(v) f(v, t) d v=: U(t)=U .
$$

This means that $P(f, f) / \mu$ is a density function and we can consider the following decomposition

$$
P(f, f)(v, t) / \mu=M[f](v, t)+g(v, t) .
$$

The function $g(v, t)$ represents the non equilibrium part of the distribution function and from the definition above it follows that $g(v, t)$ is in general non positive. Moreover since $P(f, f) / \mu$ and $M[f]$ share the first three moments we have

$$
\int_{\mathbb{R}^{d v}} \phi(v) g(v, t) d v=0 \quad \text { with } \quad \phi(v)=\left(1, v, \frac{1}{2}|v|^{2}\right) .
$$

Inserting this decomposition in (27), the Boltzmann equation can be rewritten as

$$
\partial_{t} f=\frac{\mu}{\varepsilon} g+\frac{\mu}{\varepsilon}(M[f]-f)=\frac{\mu}{\varepsilon}\left(\frac{P(f, f)}{\mu}-M[f]\right)+\frac{\mu}{\varepsilon}(M[f]-f) .
$$

Note that even if $M[f]$ is nonlinear in $f$, thanks to the conservation properties (7) of the collision operator, it remains constant in time during the relaxation process, so that we shall rename it $M[f]=: M$ in the sequel. Starting from (31), it is possible to derive an exponential integrator where we exploit the exact solution of the linear part (see [26]). Indeed, we first rewrite (31) as

$$
\frac{\partial\left((f-M) e^{\mu t / \varepsilon}\right)}{\partial t}=\frac{\mu}{\varepsilon}\left(\frac{P(f, f)}{\mu}-M\right) e^{\mu t / \varepsilon},
$$

which is obtained multiplying (31) by the integrating factor $\exp (\mu t / \varepsilon)$. A class of explicit exponential Runge-Kutta schemes is then obtained by direct application of an explicit RungeKutta method to (32). Among this class, we consider a first order in time method which we use to construct a new MC method (see [26] for high order schemes). This reads

$$
\begin{aligned}
f^{n+1} & =e^{-\frac{\mu \Delta t}{\varepsilon}} f^{n}+\frac{\mu \Delta t}{\varepsilon} e^{-\frac{\mu \Delta t}{\varepsilon}}\left(\frac{P\left(f^{n}, f^{n}\right)}{\mu}-M\right)+\left(1-e^{-\frac{\mu \Delta t}{\varepsilon}}\right) M \\
& =e^{-\frac{\mu \Delta t}{\varepsilon}} f^{n}+\frac{\mu \Delta t}{\varepsilon} e^{-\frac{\mu \Delta t}{\varepsilon}} \frac{P\left(f^{n}, f^{n}\right)}{\mu}+\left(1-e^{-\frac{\mu \Delta t}{\varepsilon}}-\frac{\mu \Delta t}{\varepsilon} e^{-\frac{\mu \Delta t}{\varepsilon}}\right) M,
\end{aligned}
$$

where the time index on $M(:=M[f])$ is omitted since this function is constant in time. One can notice from (33) that the solution in terms of the distribution function at time $t^{n+1}$ is given by a convex combination of three positive terms, independently of the choice of $\Delta t$. In particular, for $\Delta t \rightarrow+\infty$ or equivalently $\varepsilon \rightarrow 0$, one gets $f^{n+1}=M$, i.e. the distribution function is projected over the equilibrium state and then the scheme is AP according to Definition 4.1. In addition, the method is clearly unconditionally positive being a convex combination of positive quantities and one can prove that it is entropic, according to Definition 4.2. A Monte Carlo method derived from (33) can readily be obtained (see also [56]). 
Algorithm 4.1. Asymptotic Preserving Monte Carlo (APMC) method for Maxwell molecules.

- Compute the initial velocities of the particles: $\left\{v_{1}^{0}, . ., v_{N}^{0}\right\}$

- from $n=1$ to $n=n_{\text {fin }}$

- for particles from $k=1$ to $k=N$

* with probability $e^{-\frac{\mu \Delta t}{\varepsilon}}$ set $v_{k}^{n+1}=v_{k}^{n}$

* with probability $\frac{\mu \Delta t}{\varepsilon} e^{-\frac{\mu \Delta t}{\varepsilon}}$ :

a) select a random particle $j$ (whose velocity is $v_{j}^{n}$ )

b) compute $v_{k}^{\prime}$ by performing a collision between particle $k$ and particle $j$

c) assign $v_{k}^{n+1}=v_{k}^{\prime}$

* with probability $\left(1-e^{-\frac{\mu \Delta t}{\varepsilon}}-\frac{\mu \Delta t}{\varepsilon} e^{-\frac{\mu \Delta t}{\varepsilon}}\right)$, replace $v_{k}^{n}$ with a particle $v_{M, k}$ sampled

from the Maxwellian distribution $M: v_{k}^{n+1}=v_{M, k}$.

- end loop over the particles

- end loop over time

The sampling from the Maxwellian distribution, demanded by the method, can be done by standard methods such as the Box-Muller sampling method for the two dimensional Normal distribution [53]. The above scheme is not conservative as for the original Nanbu method but it conserves energy and momentum only in the mean. An alternative APMC method which is also exactly conservative at each time step is the following.

Algorithm 4.2. Conservative Asymptotic Preserving Monte Carlo (CAPMC) method for Maxwell molecules.

- Compute the initial velocities of the particles: $\left\{v_{1}^{0}, . ., v_{N}^{0}\right\}$

- from $n=1$ to $n=n_{\text {fin }}$

- set $N_{c}=\operatorname{Iround}\left(\frac{\mu \Delta t}{\varepsilon} e^{-\frac{\mu \Delta t}{\varepsilon}} N / 2\right)$ and select $N_{c}$ pairs $(i, j)$ uniformly among all possible pairs. For those pairs

a) compute $v_{i}^{\prime}$ and $v_{j}^{\prime}$ according to the collision law (3)

b) assign $v_{i}^{n+1}=v_{i}^{\prime}$ and $v_{j}^{n+1}=v_{j}^{\prime}$

- set $N_{M}=$ Iround $\left(\left(1-e^{-\frac{\mu \Delta t}{\varepsilon}}-\frac{\mu \Delta t}{\varepsilon} e^{-\frac{\mu \Delta t}{\varepsilon}}\right) N\right)$ and select $N_{M}$ particles uniformly. Denote $I_{N_{M}}$ the corresponding subset of indices so that the set of particles uniformly chosen is then $\left\{v_{k}^{n}\right\}_{k \in I_{N_{M}}}$.

a) assign $\tilde{v}_{k}=v_{M, k}$ for $k \in I_{N_{M}}$ where $v_{M, k}$ are particles sampled from the Maxwellian distribution $M$.

b) perform a moment matching of the set $\left\{\tilde{v}_{k}\right\}_{k \in I_{N_{M}}}$ to exactly match momentum and energy of the original set $\left\{v_{k}^{n}\right\}_{k \in I_{N_{M}}}$. Following [21, 10], denote $m_{1}=$ $\sum_{k \in I_{N_{M}}} v_{k}^{n}$ and $m_{2}=\sum_{k \in I_{N_{M}}}\left|v_{k}^{n}\right|^{2}$ the first two moments of the original set 
and denote $\tilde{m}_{1}=\sum_{k \in I_{N_{M}}} \tilde{v}_{k}$ and $\tilde{m}_{2}=\sum_{k \in I_{N_{M}}}\left|\tilde{v}_{k}\right|^{2}$ the first two moments that come from the Maxwellian sampling. Then

$$
v_{k}^{\prime}=\left(\tilde{v}_{k}-\tilde{m}_{1}\right) / c+m_{1}, k \in I_{N_{M}} \quad \text { with } \quad c=\sqrt{\frac{m_{2}-m_{1}^{2}}{\tilde{m}_{2}-\tilde{m}_{1}^{2}}}
$$

c) Set $v_{k}^{n+1}=v_{k}^{\prime}$ for $k \in I_{N_{M}}$.

- set $v_{k}^{n+1}=v_{k}^{n}$ for all other particles which have not collided or have not be replaced by Maxwellian samples.

- end loop over time

We remark that the moment correction procedure described in the case of Maxwellian samples should be used with caution. In fact, the new samples $\left\{v_{k}^{n+1}\right\}_{k \in I_{N_{M}}}$ are no longer independent and thus the Central Limit Theorem does not apply directly, see [10] for details.

\section{A new Asymptotic Preserving Monte Carlo method}

The APMC and the CAPMC methods described in the previous Section permit to overcome the stability restrictions induced by standard explicit schemes and they avoid that the number of collisions grows to infinity if the collisional scale becomes very high. However, these methods do not solve the problem related to the statistical error. In fact, even in the limit, when the solution is analytically known, the MC methods continue to perform collisions and this does not decrease the statistical error. Since the computational cost is a primary issue and the error scales with the square root of the number of samples, in this Section, we discuss a remedy based on a new MC method for the perturbation function $g(v, t)$ instead that for $f(v, t)$, where $f$ and $g$ are linked by the following decomposition $f(v, t)=M(v)+g(v, t)$. This decomposition has already been exploited to design efficient deterministic numerical schemes for nonlinear collision operator in several papers as [32, 43]. Indeed, our goal is to design a class of schemes which avoids the resolution of the small scale dynamics induced by the particle interactions and whose cost diminishes as the equilibrium state is approached. Thus, the following requests should be fulfilled by this new MC method:

- The statistical error must be smaller than the one of standard Monte Carlo schemes.

- The collisional scale must not impose time steps limitations.

- The computational cost must decrease as the equilibrium state is approached. At the same time, the variance should diminish as the number of interactions increases.

In the following we stick to the particular case of interaction laws for which the resulting Boltzmann equation can be written as (27). Extensions to more general cases are the subject of future investigations. For this equation, the first step consists in writing the distribution function $f$ according to the following micro-macro decomposition $f(v, t)=M(v)+g(v, t)$. Now, since 
$M(v)(:=M[f](v))$ and $f(v, t)$ shares the same first three moments, we have $\int_{\mathbb{R}^{d}} \phi(v) g(v, t) d v=$ 0 . We rewrite $(32)$ using this new variable $g(v, t)$ as

$$
\begin{aligned}
\frac{\partial\left(g e^{\mu t / \varepsilon}\right)}{\partial t} & =\frac{1}{\varepsilon}(P(M+g, M+g)-\mu M) e^{\mu t / \varepsilon} \\
& =\frac{1}{\varepsilon}(P(M, M)+P(g, g)+P(M, g)+P(g, M)-\mu M) e^{\mu t / \varepsilon}
\end{aligned}
$$

where we used the bilinearity property of the gain operator $P$. Now, by noticing that $P(M, M)=$ $\mu M$, we finally have

$$
\frac{\partial\left(g e^{\mu t / \varepsilon}\right)}{\partial t}=\frac{1}{\varepsilon}(P(g, g)+P(M, g)+P(g, M)) e^{\mu t / \varepsilon} .
$$

We are now ready to derive an exponential Runge-Kutta method for (35). This is done as for the case of (32) by applying a first order explicit Runge-Kutta method to (35). This reads

$$
g^{n+1}=e^{-\frac{\mu \Delta t}{\varepsilon}} g^{n}+\frac{\mu \Delta t}{\varepsilon} e^{-\frac{\mu \Delta t}{\varepsilon}}\left(\frac{P\left(g^{n}, g^{n}\right)+P\left(g^{n}, M\right)+P\left(M, g^{n}\right)}{\mu}\right) .
$$

Observe that now, contrary to (33), (36) is not a convex combination of positive functions anymore. In particular, since $\left(e^{-\frac{\mu \Delta t}{\varepsilon}}+\frac{\mu \Delta t}{\varepsilon} e^{-\frac{\mu \Delta t}{\varepsilon}}\right) \leq 1$ for every choice of the time step $\Delta t$, we have $g \rightarrow 0$ exponentially fast when $\Delta t \rightarrow+\infty$ or $\varepsilon \rightarrow 0$, which implies that $f(v, t)$ is projected over the equilibrium state, i.e. the AP property is verified. This interesting property can be exploited in the construction of a new Monte Carlo method. Before introducing our new MC method, we still need one passage which consists in dividing the perturbation $g$ into a difference of two positive parts: $g(v, t)=g_{p}(v, t)-g_{m}(v, t)$, where $g_{p}(v, t):=\max (g(v, t), 0)$ and $g_{m}(v, t):=-\min (g(v, t), 0)$. In the above decomposition, both parts are positive, $g_{p}(v, t) \geq 0$ and $g_{m}(v, t) \geq 0, \forall v \in \mathbb{R}^{d_{v}}$ and consequently they can then be reinterpreted as probability distributions once suitably normalized. Now, (36) can be rewritten as

$$
\begin{aligned}
g_{p}^{n+1}-g_{m}^{n+1}= & e^{-\frac{\mu \Delta t}{\varepsilon}}\left(g_{p}^{n}-g_{m}^{n}\right)+\frac{\mu \Delta t}{\varepsilon} e^{-\frac{\mu \Delta t}{\varepsilon}}\left(\frac{P\left(g_{p}^{n}, g_{p}^{n}\right)+P\left(g_{p}^{n}, M\right)+P\left(M, g_{p}^{n}\right)+P\left(g_{m}^{n}, g_{m}^{n}\right)}{\mu}\right) \\
& -\frac{\mu \Delta t}{\varepsilon} e^{-\frac{\mu \Delta t}{\varepsilon}}\left(\frac{P\left(g_{p}^{n}, g_{m}^{n}\right)+P\left(g_{m}^{n}, M\right)+P\left(M, g_{m}^{n}\right)+P\left(g_{m}^{n}, g_{p}^{n}\right)}{\mu}\right) .
\end{aligned}
$$

Then, since $P$ is positive, one deduces the equations for the $g_{p}^{n+1}$ and $g_{m}^{n+1}$

$$
g_{p}^{n+1}=e^{-\frac{\mu \Delta t}{\varepsilon}} g_{p}^{n}+\frac{\mu \Delta t}{\varepsilon} e^{-\frac{\mu \Delta t}{\varepsilon}}\left(\frac{P\left(g_{p}^{n}, g_{p}^{n}\right)+P\left(g_{p}^{n}, M\right)+P\left(M, g_{p}^{n}\right)+P\left(g_{m}^{n}, g_{m}^{n}\right)}{\mu}\right),
$$

and

$$
g_{m}^{n+1}=e^{-\frac{\mu \Delta t}{\varepsilon}} g_{m}^{n}+\frac{\mu \Delta t}{\varepsilon} e^{-\frac{\mu \Delta t}{\varepsilon}}\left(\frac{P\left(g_{p}^{n}, g_{m}^{n}\right)+P\left(g_{m}^{n}, M\right)+P\left(M, g_{m}^{n}\right)+P\left(g_{m}^{n}, g_{p}^{n}\right)}{\mu}\right) .
$$

We are now in the same setting in which the Monte Carlo and the APMC methods have been derived. In particular due to the structure of the operator $P$ in the case of Maxwellian molecules, 
the sampling from $P(h, \ell)$ with $h$ and $\ell$ two positive functions can be performed by applying the collision law (3) to a particle from $h$ with a random particle from $\ell$.

We now approximate the two distributions $g_{p}(v, t)$ and $g_{m}(v, t)$ by a finite set of $N_{p}(t)$ and $N_{m}(t)$ particles whose velocity is $v_{p, k}(t)$ and $v_{m, k}(t)$ (as we shall see, the number of particles will change with time)

$$
\begin{gathered}
g_{p}(v, t) \approx m \sum_{k=1}^{N_{p}(t)} \delta_{v_{p, k}(t)}(v), \\
g_{m}(v, t) \approx m \sum_{k=1}^{N_{m}(t)} \delta_{v_{m, k}(t)}(v),
\end{gathered}
$$

where the weights $m$ are constant

$$
m=\frac{1}{N_{p}(t=0)} \int_{\mathbb{R}^{d v}} g_{p}(v, t=0) d v=\frac{1}{N_{m}(t=0)} \int_{\mathbb{R}^{d_{v}}} g_{m}(v, t=0) d v .
$$

Indeed, noticing that $\int_{\mathbb{R}^{d v}}\left(g_{p}(v, t)-g_{m}(v, t)\right) d v=0, \forall t$, one should have that $N_{p}=N_{m}$ in average. In fact, this is strictly imposed at $t=0$ by the initial sampling procedure and thus the weights for positive and negative particles are equal (and both equal to $m$ ). Concerning, the number of samples from the two distinct distributions, these are also equal at the beginning $N_{p}(t=0)=N_{m}(t=0)$ and they remain in the mean (but not necessarily exactly) equal during the time evolution.

Instead of considering a Monte Carlo method for equations (38) and (39) which will be computationally inefficient and will cause the creation of unnecessary new samples as made precise later, we use (36) for the construction of our new MC method. To that aim, let be given the set of particles velocities $I_{g}:=\left\{v_{p, k}(t)\right\}_{k=1, \ldots, N_{p}(t)} \cup\left\{v_{m, \ell}(t)\right\}_{\ell=1, \ldots, N_{m}(t)}$ used to approximate $g(v, t)$, this corresponds to the union of samples from $g_{p}(v, t)$ and from $g_{m}(v, t)$. Then, the sampling of $P(g, g)$ in (36) is performed as follows. One selects a pair of particles $(i, j)$ whose velocities are $v_{i}, v_{j} \in I_{g}$ and computes the post-collisional velocity $v_{i}^{\prime}$ according to (3) for this collision. Then, the decision whether the new velocity $v_{i}^{\prime}$ belongs to the positive or negative part of $g(v, t)$ lies in equations (38)-(39): if $v_{i}$ and $v_{j}$ belongs both to the positive category (resp. negative), this means that we are approximating $P\left(g_{p}, g_{p}\right)$ (resp. $P\left(g_{m}, g_{m}\right)$ ) and the new velocity $v_{i}^{\prime}$ then belongs to the positive category according to (38). On the contrary, if $v_{i}$ and $v_{j}$ belongs to two different categories, the resulting velocity $v_{i}^{\prime}$ then belongs to the negative category according to (39). This permits to aggregate the four contributions $P\left(g_{p}, g_{p}\right), P\left(g_{p}, g_{m}\right), P\left(g_{m}, g_{p}\right)$ and $P\left(g_{m}, g_{m}\right)$ in a sole step. The MC method based on the previous tools is called APMCG and is presented in the following algorithm.

Algorithm 5.1. Asymptotic Preserving Monte Carlo (APMCG) method for Maxwell molecules.

- Compute the initial velocities of the particles belonging to the approximation of $g_{p}(v, t=0)$ and $g_{m}(v, t=0):\left\{v_{p, 1}^{0}, . ., v_{p, N_{p}(0)}^{0}\right\},\left\{v_{m, 1}^{0}, . ., v_{m, N_{m}(0)}^{0}\right\}$.

- from $n=1$ to $n=n_{\text {fin }}$

- set $N K_{p}=\operatorname{Iround}\left(e^{-\frac{\mu \Delta t}{\varepsilon}} N_{p}(n \Delta t)\right)$ and select $N K_{p}$ particles uniformly. Denote $I_{p}$ the corresponding subset of indices. Set $v_{p, k}^{n+1}=v_{p, k}^{n}$ for $k \in I_{p}$. 
- set $N K_{m}=\operatorname{Iround}\left(e^{-\frac{\mu \Delta t}{\varepsilon}} N_{m}(n \Delta t)\right)$ and select $N K_{m}$ particles uniformly. Denote $I_{m}$ the corresponding subset of indices. Set $v_{m, k}^{n+1}=v_{m, k}^{n}$ for $k \in I_{m}$.

- discard a random fraction $N D_{p}=\operatorname{Iround}\left(\left(1-e^{-\frac{\mu \Delta t}{\varepsilon}}-\frac{\mu \Delta t}{\varepsilon} e^{-\frac{\mu \Delta t}{\varepsilon}}\right) N_{p}(n \Delta t)\right)$.

- discard a random fraction $N D_{m}=\operatorname{Iround}\left(\left(1-e^{-\frac{\mu \Delta t}{\varepsilon}}-\frac{\mu \Delta t}{\varepsilon} e^{-\frac{\mu \Delta t}{\varepsilon}}\right) N_{m}(n \Delta t)\right)$.

- sampling of $P(g, g) / \mu_{g}$ : keep a fraction $N C_{1}=$ Iround $\left(\frac{\mu_{g} \Delta t}{\varepsilon} e^{-\frac{\mu \Delta t}{\varepsilon}}\left(N_{p}(n \Delta t)+N_{m}(n \Delta t)\right)\right)$. Denote $I_{c 1}$ the random set (of size $N C_{1}$ ) of particles indices and $S c 1=\left\{v_{k}^{n}\right\}_{k \in I_{c 1}}$ the set composed of positive or negative particles.

a) compute $v_{k}^{\prime}$ by performing a collision between a particle $k$ and a random particle $j\left(k, j \in I_{c 1}\right)$.

b) assign $v_{p, k}^{n+1}=v_{k}^{\prime} \quad$ if both particles belong to the same category (positive or negative), $v_{m, k}^{n+1}=v_{k}^{\prime} \quad$ if the particles do not belong to the same category.

- sampling of $P(g, M) / \mu$ : keep a fraction $N C_{2}=\operatorname{Iround}\left(\frac{\mu \Delta t}{\varepsilon} e^{-\frac{\mu \Delta t}{\varepsilon}}\left(N_{p}(n \Delta t)+N_{m}(n \Delta t)\right)\right)$. Denote $I_{c 2}$ the random set (of size $N C_{2}$ ) of particles indices and $S c 2=\left\{v_{k}^{n}\right\}_{k \in I_{c 2}}$ the set composed of positive or negative particles.

a) compute $v_{k}^{\prime}$ by performing a collision between a particle $k \in I_{c 2}$ and a random particle $j$ sampled from the Maxwellian distribution $M$, whose velocity is $v_{M, j}$.

b) assign $v_{p, k}^{n+1}=v_{k}^{\prime} \quad$ if the particle $k$ belongs to the positive category, $v_{m, k}^{n+1}=v_{k}^{\prime} \quad$ if the particle $k$ belongs to the negative category.

- sampling of $P(M, g) / \mu$ : keep a fraction $N C_{3}=\operatorname{Iround}\left(\frac{\mu \Delta t}{\varepsilon} e^{-\frac{\mu \Delta t}{\varepsilon}}\left(N_{p}(n \Delta t)+N_{g}(n \Delta t)\right)\right)$. Denote $I_{c 3}$ the random set (of size $N C_{3}$ ) of particles indices and $S c 3=\left\{v_{k}^{n}\right\}_{k \in I_{c 3}}$ the set composed of positive or negative particles.

a) compute $v_{k}^{\prime}$ by performing a collision between the particle with velocity $v_{M, k}$ and a random particle $j$ whose velocity $v_{j}^{n} \in S_{c 3}$.

b) assign $v_{p, k}^{n+1}=v_{k}^{\prime} \quad$ if the random particle $j$ belonged to the positive category, $v_{m, k}^{n+1}=v_{k}^{\prime} \quad$ if the random particle $j$ belonged to the negative category.

- end loop over time

Remark 1. The above described method deserves some remarks.

- The factor $\mu_{g}$ in the expression for $N C_{1}$ is a normalization factor which comes from the fact that in (38) and (39) the collision operators are divided by the constant $\mu$ which was used to normalize the original operator $P(f, f)$. The constant $\mu_{g}$ takes the value

$$
\mu_{g}(t)=\int_{\mathbb{R}^{d_{v}}} \int_{\mathbb{S}^{d_{v}-1}} b(\omega)\left(g_{p}(v, t)-g_{m}(v, t)\right) d v d \omega
$$


- The method is not conservative in the sense that the number of particles belonging to the distribution $g_{p}^{n+1}(v)$ in general is not equal to the number of particles belonging to the distribution $g_{m}^{n+1}(v)$

$$
N_{p}((n+1) \Delta t) \neq N_{m}((n+1) \Delta t) .
$$

The same holds true for the first order moment

$$
\int_{\mathbb{R}^{d v}} v g_{p}^{n+1}(v) d v \neq \int_{\mathbb{R}^{d_{v}}} v g_{m}^{n+1}(v) d v
$$

and the second order one

$$
\int_{\mathbb{R}^{d_{v}}}|v|^{2} g_{p}^{n+1}(v) d v \neq \int_{\mathbb{R}^{d_{v}}}|v|^{2} g_{m}^{n+1}(v) d v
$$

Conservations are indeed verified only in the mean but not at each collision.

In the sequel, we discuss the effect of the new strategy on the number of particles. Indeed, as mentioned in the Introduction, one major problem in considering the decomposition $f=M+g$ lies in the fact that the total number of particles may increase (see $[59,60,42])$. In order to shed some lights on this fact, let denote by $N_{g}(t):=N_{p}(t)+N_{m}(t)$ the total number of particles used to sample $g(v, t)=g_{p}(v, t)-g_{m}(v, t)$ at time $t$. Then, the collisional step causes the introduction of $N C:=N C_{1}+N C_{2}+N C_{3}$ particles (where $N C_{1}, N C_{2}$ and $N C_{3}$ are integers defined in Algorithm 5.1)

$$
N C=\text { Iround }\left(\frac{\mu \Delta t}{\varepsilon} e^{-\frac{\mu \Delta t}{\varepsilon}} N_{g}(t)\left(2+\mu_{g} / \mu\right)\right) .
$$

The total number of particles after one time step is consequently given by

$$
N_{g}(t+\Delta t)=\text { Iround }\left(e^{-\frac{\mu \Delta t}{\varepsilon}} N_{g}(t)\right)+N C .
$$

This means that the total number of samples may increase after one time step. Indeed, the condition which guarantees that $N_{g}(t+\Delta t) \leq N_{g}(t)$ is

$$
\operatorname{IF}(\mu \Delta t / \varepsilon):=\left(e^{-\frac{\mu \Delta t}{\varepsilon}}+\left(2+\mu_{g} / \mu\right) \frac{\mu \Delta t}{\varepsilon} e^{-\frac{\mu \Delta t}{\varepsilon}}\right) \leq 1,
$$

where typically $\mu_{g} / \mu \leq 1$ even when the system is far from equilibrium.

Figure 1 shows the increasing factor function $\operatorname{IF}(\mu \Delta t / \varepsilon)$ given by (45) for different values of $\mu \Delta t / \varepsilon \in[0,2]$ and in two different configurations: $\mu_{g} / \mu=1$ (worst scenario i.e. the system is far from equilibrium) and $\mu_{g} / \mu=0$ (best scenario i.e. the system is close to equilibrium). We also display in Figure 1 the $I F$ function in the case of the Algorithms 4.1 and 4.2 (in this case $\operatorname{IF}(x)=e^{-x}(1+x)$ with $\left.x=\mu \Delta t / \varepsilon\right)$. For the Algorithms 4.1 and 4.2, the $I F$ is always less than one since the solution is a convex combination of positive quantities. The optimal configuration for the APMCG method would correspond to this latter case of $I F$. Instead, from Figure 1, it is clear that, in general, we should expect that the number of samples increases with time. However, as already observed $g$ tends to zero exponentially fast as we approach the equilibrium state. This means that, the APMCG method described in Algorithm 5.1 introduces 


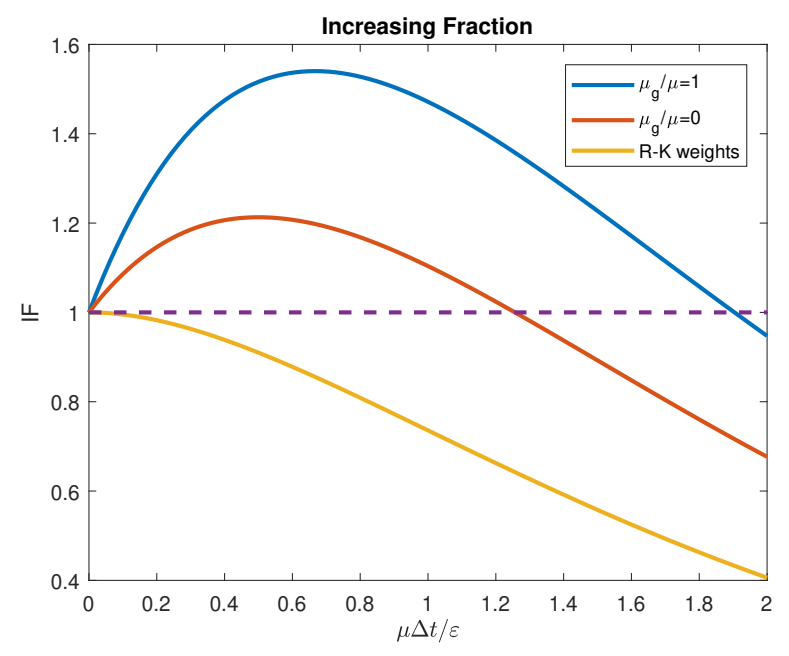

Figure 1: Increasing Factor $(I F)$ defined in (45) for different values of $\mu_{g} / \mu$. The $I F$ in the case of the original weights of the Runge-Kutta exponential scheme (33) (i.e. $\operatorname{IF}(x)=e^{-x}(1+x), x=$ $\mu \Delta t / \varepsilon)$ is also reported.

redundant information into the system, i.e. there exist particles belonging to $g_{p}(v, t)$ and to $g_{m}(v, t)$ furnishing the same information with opposite sign.

A solution to this problem consists in eliminating, at least, a number of particles such that $N_{g}(t+\Delta t) \leq N_{g}(t)$. This means that a fraction $\alpha(\mu \Delta t / \varepsilon)$ of $N C$ (defined by (43)) has to be eliminated. This fraction is computed such that $e^{-\frac{\mu \Delta t}{\varepsilon}}+(1-\alpha(\mu \Delta t / \varepsilon)) N C \leq 1$ which means

$$
\alpha(\mu \Delta t / \varepsilon) \geq \max \left(0,1-\frac{1-e^{-\frac{\mu \Delta t}{\varepsilon}}}{\left(2+\mu_{g} / \mu\right) \frac{\mu \Delta t}{\varepsilon} e^{-\frac{\mu \Delta t}{\varepsilon}}}\right) .
$$

In Figure 2, the function $\alpha$ given by (46) is plotted as a function of $\mu \Delta t / \varepsilon$ for two different values of the ratio $\mu_{g} / \mu$. The main issue consists then in finding a method which reduces the number of samples at a cost which is linear or close to linear with respect to the number of particles used to sample $g(v, t)$. To that aim, we perform a density kernel estimate (KDE) procedure which employs only a random subset of the set of the particles created by the collision step (whose number is $N C$ ). This density estimate is then used in an acceptance-rejection technique [20] to decide which samples can be eliminated without losing information in the solution $g(v, t)$. Let observe that even if, at a first sight, this KDE procedure may seem costly, this is not the case in practice. Indeed, the number of particles belonging to the distribution $g(v, t)$ decays in time and they are always less than the number of particles one can use in a standard MC method maintaining equivalent accuracy, i.e. with an equivalent weight $m$. Moreover, we only consider a fraction of this number for performing the KDE. The KDE procedure works in the following way. Given a set of $\tilde{N}_{p}(t)$ (resp. $\tilde{N}_{m}(t)$ ) samples after the collision step (i.e. $\tilde{N}_{p}(t)+\tilde{N}_{m}(t)=N C$ ) from $g_{p}(v, t)$ (resp. $g_{m}(v, t)$ ) and the corresponding fractions $\hat{N}_{p}(t)$ (resp. 


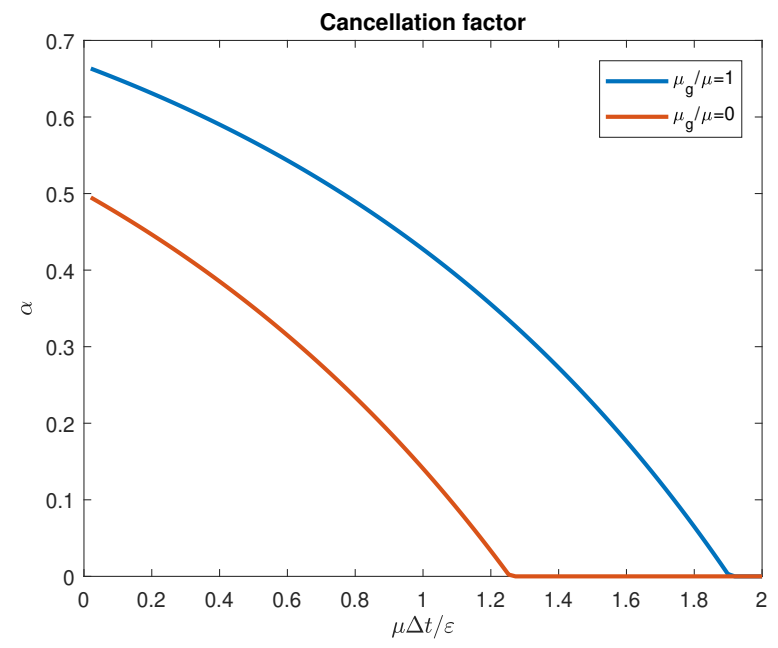

Figure 2: Cancellation factor $\alpha$ given by (46) as a function of $\mu \Delta t / \varepsilon$ for $\mu_{g} / \mu=1$ and $\mu_{g} / \mu=0$.

$\left.\hat{N}_{m}(t)\right)$, one estimates the distribution $g_{p}\left(\right.$ resp. $\left.g_{m}\right)$ by $\hat{g}_{p}\left(\right.$ resp. $\left.\hat{g}_{m}\right)$

$$
\hat{g}_{p}(v, t)=\frac{1}{\hat{N}_{p}(t) h^{d_{v}}} \sum_{k=1}^{\hat{N}_{p}(t)} K\left(\frac{v-v_{p, k}(t)}{h}\right)
$$

respectively

$$
\hat{g}_{m}(v, t)=\frac{1}{\hat{N}_{m}(t) h^{d_{v}}} \sum_{k=1}^{\hat{N}_{m}(t)} K\left(\frac{v-v_{m, k}(t)}{h}\right),
$$

where $K(v)$ is the kernel having the following properties

$$
K(v)=K(-v) \forall v \in \mathbb{R}^{d_{v}}, \quad \int_{\mathbb{R}^{d v}} K(v) d v=1, \quad \lim _{v \rightarrow \pm \infty=0} K(v)=0 .
$$

In particular, we choose a Gaussian kernel $K(v)=\frac{1}{(2 \pi)^{d v / 2}} \exp \left(-|v|^{2} / 2\right)$. Let us remark that other choices are possible, such that splines shape functions. Here, the choice of the bandwidth $h$ is done in such a way to minimize the mean integrated square error $\left(M I S E_{h}\right)$

$$
M I S E_{h}(t)=\int_{\mathbb{R}^{d_{v}}} \mathbb{E}\left[\left(g_{p}(v, t)-\hat{g}_{p}(v, t)\right)^{2}\right] d v
$$

with $h>0, \mathbb{E}$ the expectation and where we assume that the unknown function is normally distributed, which is the case as the number of collision grows due to the collisional effect. This gives

$$
h_{o p t}=\left(\frac{4 \mu_{K}}{\frac{\sigma_{K}^{4}}{N} \int\left|\nabla^{2} f(v)\right|^{2} d v}\right)^{1 /\left(4+d_{v}\right)}
$$

with $f(v)$ the normal distribution, $N$ the number of samples $\left(N=\hat{N}_{p}(t)\right.$ or $\left.N=\hat{N}_{m}(t)\right)$, $\sigma_{K}^{2}=\int_{\mathbb{R}^{d_{v}}}|v|^{2} K(v) d v, \mu_{K}=\int_{\mathbb{R}^{d_{v}}} K^{2}(v) d v$. Once $\hat{g}_{p}(v, t)$ and $\hat{g}_{m}(v, t)$ have been estimated one 
computes the estimation of the distribution function

$$
\hat{g}(v, t)=\hat{g}_{p}(v, t)-\hat{g}_{m}(v, t) .
$$

Thanks to this estimation one can finally decide to keep or not a sample by an acceptancerejection technique. The algorithm works as follow

Algorithm 5.2. Acceptance-Rejection method for the APMCG scheme.

- Let $\tilde{N}_{p}(t)$ respectively $\tilde{N}_{m}(t)$ be the positive and the negative particles of the set composed by $N C$, the number of particles after the collision step.

- Given the velocities $\left\{v_{p, k}(t)\right\}_{k=1, \ldots, \tilde{N}_{p}(t)}$ (resp. $\left.\left\{v_{m, k}(t)\right\}_{k=1, \ldots, \tilde{N}_{m}(t)}\right)$ of the particles belonging to the approximation of $g_{p}(v, t)$ (resp. $g_{m}(v, t)$ ) after the collision step and given the KDE estimation of $\hat{g}_{p}(v, t), \hat{g}_{m}(v, t)$ and $\hat{g}(v, t)$.

- from $k=1$ to $k=\tilde{N}_{p}(t)$

- if $\hat{g}\left(v_{p, k}(t), t\right) / \hat{g}_{p}\left(v_{p, k}(t), t\right)>\xi$ with $\xi$ a random number in $[0,1]$ keep the particle.

- otherwise discard it.

- from $k=1$ to $k=\tilde{N}_{m}(t)$

- if $\hat{g}\left(v_{m, k}(t), t\right) / \hat{g}_{m}\left(v_{m, k}(t), t\right)>\xi$ with $\xi$ a random number in $[0,1]$ keep the particle.

- otherwise discard it.

\section{$6 \quad$ Numerical results}

In this Section, we discuss the different aspects of the APMCG method described in the previous part. The discussion and the analysis is divided in several parts. We start by analyzing the different collisional operators appearing in the Monte Carlo method detailed in 5.1 in comparison with the solution furnished by the spectral method briefly detailed in the Appendix A. Then, we study the problem related to the increasing number of samples needed to compute the collisional part in the APMCG method and the remedy that has been described to overcome this issue (Kernel Density Estimation and Acceptance-Rejection technique). Successively, we analyze the solution produced by the new method (the one described in Algorithm 5.1 combined with the discarding part) by comparing it with a standard $\mathrm{MC}$ solution and again with the spectral method. In this case, the errors produced by the MC and the APMCG are studied and measurements of the efficiency of the two MC based schemes are reported. We consider, in the following tests, the Boltzmann equation with Maxwellian molecules and a two dimensional velocity space $\left(d_{v}=2\right)$.

\subsection{Collisional terms analysis}

We start by analyzing the three collisional terms of the Algorithm 5.1 separately. The idea is to show that each single term is well described by our Monte Carlo approach. Namely, we consider $P\left(g_{p}-g_{m}, g_{p}-g_{m}\right), P\left(g_{p}-g_{m}, M\right)$ and $P\left(M, g_{p}-g_{m}\right)$ and we compare the solution obtained by 
the APMCG with a solution obtained with a reference solution (given by the spectral method described in the Appendix A).

The initial data is the following

$$
f(v, t=0)= \begin{cases}0.25, & \text { if } v:=\left(v_{x}, v_{y}\right) \in \mathcal{D}=\left\{\left(v_{x}, v_{y}\right) \in \mathbb{R}^{2}: v_{x}^{2}+v_{y}^{2} \leq 1\right\} \\ 0, & \text { if } v:=\left(v_{x}, v_{y}\right) \in \mathbb{R}^{2} \backslash \mathcal{D}\end{cases}
$$

The velocity domain needed to compute the solution of the collision terms by means of spectral methods is truncated taking $f(v, t) \in \mathcal{C}$ with $\mathcal{C}=[-8,8]^{2}, N_{v}=128$ points in each direction. Concerning the Monte Carlo method, we choose a number of particles $N=10^{6}$ to sample the initial distribution $f(v, t=0)$ (see Figure 3 for its shape). With the above choices and thanks to the micro-macro decomposition $g(v, t=0)=f(v, t=0)-M(v)$, we obtain that the number of particles representing the perturbation $g(v, t=0)$ is $N_{g} \approx 6 \times 10^{5}$. Figure 3 reports the shape of $g(v, t)$ and of the Maxwellian equilibrium $M(v)$ on the same grid as the one used for the spectral method. These three quantities are used to compute the three collisional integrals mentioned above. Figures from 4 to 6 report, to that aim, some plots relative to $P\left(g_{p}-g_{m}, g_{p}-g_{m}\right)$, $P\left(g_{p}-g_{m}, M\right)$ and $P\left(M, g_{p}-g_{m}\right)$. In each Figure, we show, for each single term and using the APMCG and spectral methods, the two-dimensional representation of the solution (i.e. as a function of $\left.v:=\left(v_{x}, v_{y}\right)\right)$ and two one dimensional slices $\left(v_{y}=0\right.$ is fixed and $v_{x}=0$ is fixed). For APMCG, the collision elements once computed are then reconstructed by histograms over the same grid on which the spectral method lives. We clearly see that the APMCG approach well describes the three operators. Then, in Figures 7 and 8, we finally report the results obtained when the three collisional operators are summed up:

$$
\tilde{g}(v):=P\left(g_{p}-g_{m}, g_{p}-g_{m}\right)(v)+P\left(g_{p}-g_{m}, M\right)(v)+P\left(M, g_{p}-g_{m}\right)(v) .
$$

In more details, Figure 7 shows the comparisons between the spectral and the APMCG methods on the slices (first with $v_{y}=0$ and then with $v_{x}=0$ ). Figure 8 shows instead the full twodimensional representation obtained by the two methods (spectral and APMCG) as well as their pointwise error. We can conclude that the new APMCG strategy is able to capture in a good way this part of the solution.

\subsection{Analysis of the Increasing Factor $I F$ function}

Once verified that the APMCG method is able to well describe the collision operator, we analyze now the role played by the number of particles in the results. As explained in the previous Section, the number of particles required to sample the three terms $P\left(g_{p}-g_{m}, g_{p}-g_{m}\right), P\left(g_{p}-\right.$ $\left.g_{m}, M\right)$ and $P\left(M, g_{p}-g_{m}\right)$ which appears in the collision step of Algorithm 5.1 is larger than the number of particles used to sample $g_{p}$ and $g_{m}$. However, all the new created samples are not strictly necessary to approximate the solution. This redundant information can consequently be cancelled without losing precision in the approximation of the solution. To that aim, we now

show, in Figure 9, the positive part $\tilde{g}_{p}(v)$ and the negative part $\tilde{g}_{m}(v)$ of these three operators using our APMCG strategy. From these two functions, one can reconstruct $\tilde{g}(v)=\tilde{g}_{p}(v)-\tilde{g}_{m}(v)$. It is clear from Figure 9 that even if the difference $\tilde{g}(v)=\tilde{g}_{p}(v)-\tilde{g}_{m}(v)$ is well captured, each distribution $\tilde{g}_{p}(v)$ and $\tilde{g}_{m}(v)$ is such that:

$$
\tilde{g}_{p}(v) \geq \max (\tilde{g}(v), 0), \quad \tilde{g}_{m}(v) \leq-\min (\tilde{g}(v), 0) .
$$



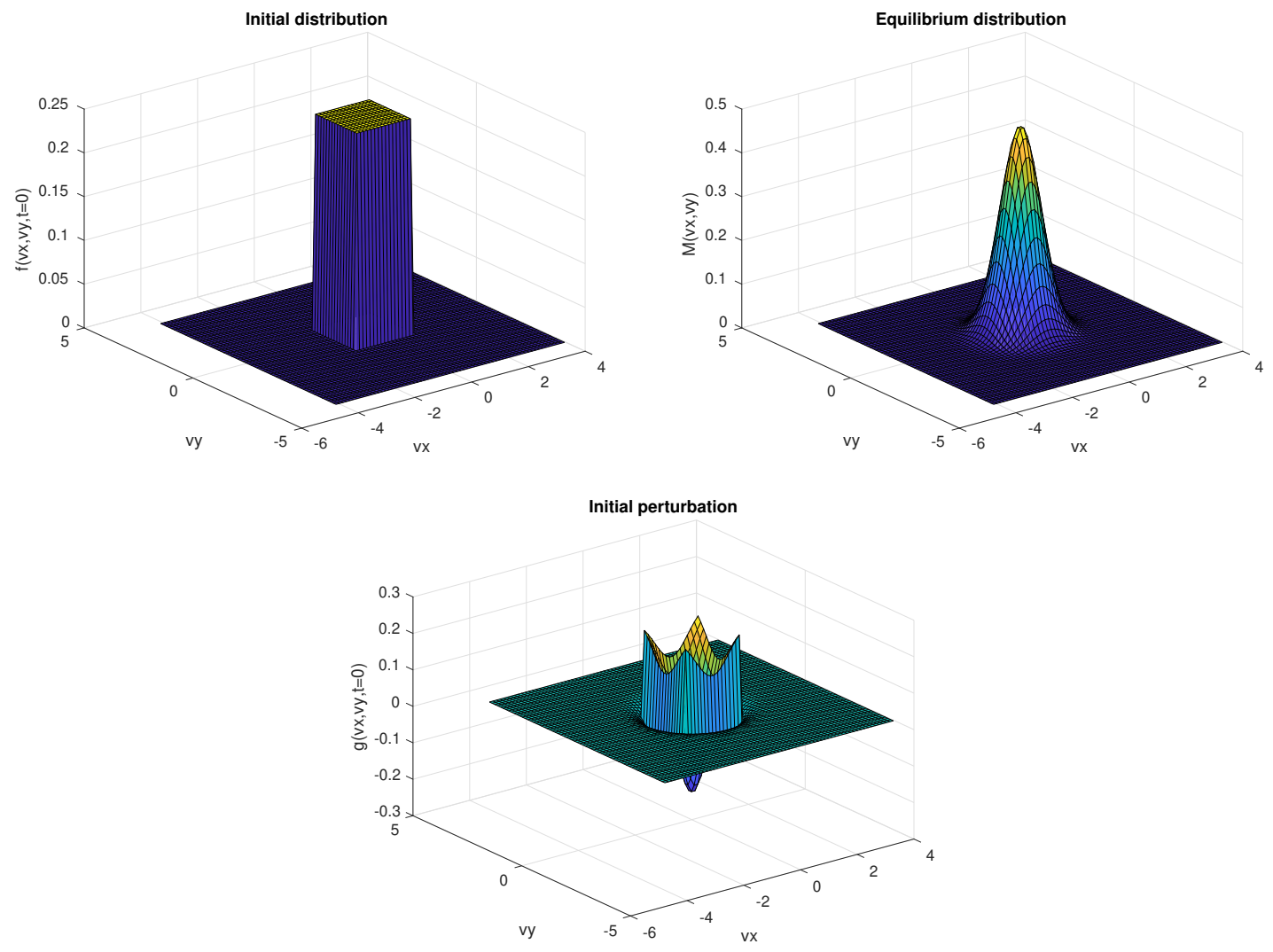

Figure 3: Top left: $f(v, t=0)$. Top right: $M(v)$. Bottom: $g(v, t=0)$.

As a result, we are considering a larger set of samples than needed. In order to solve this problem, we introduce then the KDE and the acceptance/rejection techniques. In Figure 10, we report the two dimensional functions $\hat{g}_{p}(v)$ and $\hat{g}_{m}(v)$ that are obtained using KDE and acceptance/rejection from $\tilde{g}_{p}(v)$ and $\tilde{g}_{m}(v)$ (see Algorithm 5.2) together with their slices (for $v_{y}=0$ and $\left.v_{x}=0\right)$. We denote $\hat{g}(v)=\hat{g}_{p}(v)-\hat{g}_{m}(v)$ the resulting function. Here we have $N C=1.5 \times 10^{6}$ after the collision step and we used $N C / 10$ particles to reconstruct $\hat{g}(v)$ using the KDE technique. Then, after the acceptance/rejection step, we finally get $2.7 \times 10^{5}$ particles to sample $\tilde{g}$, which means that the number of particles has been divided by more than 5 (i.e. $\left.1.5 \times 10^{6} / 2.7 \times 10^{5}\right)$.

As observed in Figure 10, the error (with respect to the spectral method) is equivalent to the error shown in Figure 10 without the elimination step which confirms the very good behavior of the new method. Finally, Figure 11 shows the $L^{1}$ norm of the error between the spectral calculation of $\tilde{g}(v)$ (which is assumed to be the reference solution) and the new APMCG method by considering different values for $N_{K D E}$ ranging from $2 \times 10^{4}$ to $10^{6}$ and for different values of the bandwidth $h$. The case $N_{K D E}=10^{6}$ corresponds to the case in which all particles are used to reconstruct the distribution $\hat{g}(v)$. As expected, the optimal bandwidth formula (51) is the one giving the best results. 

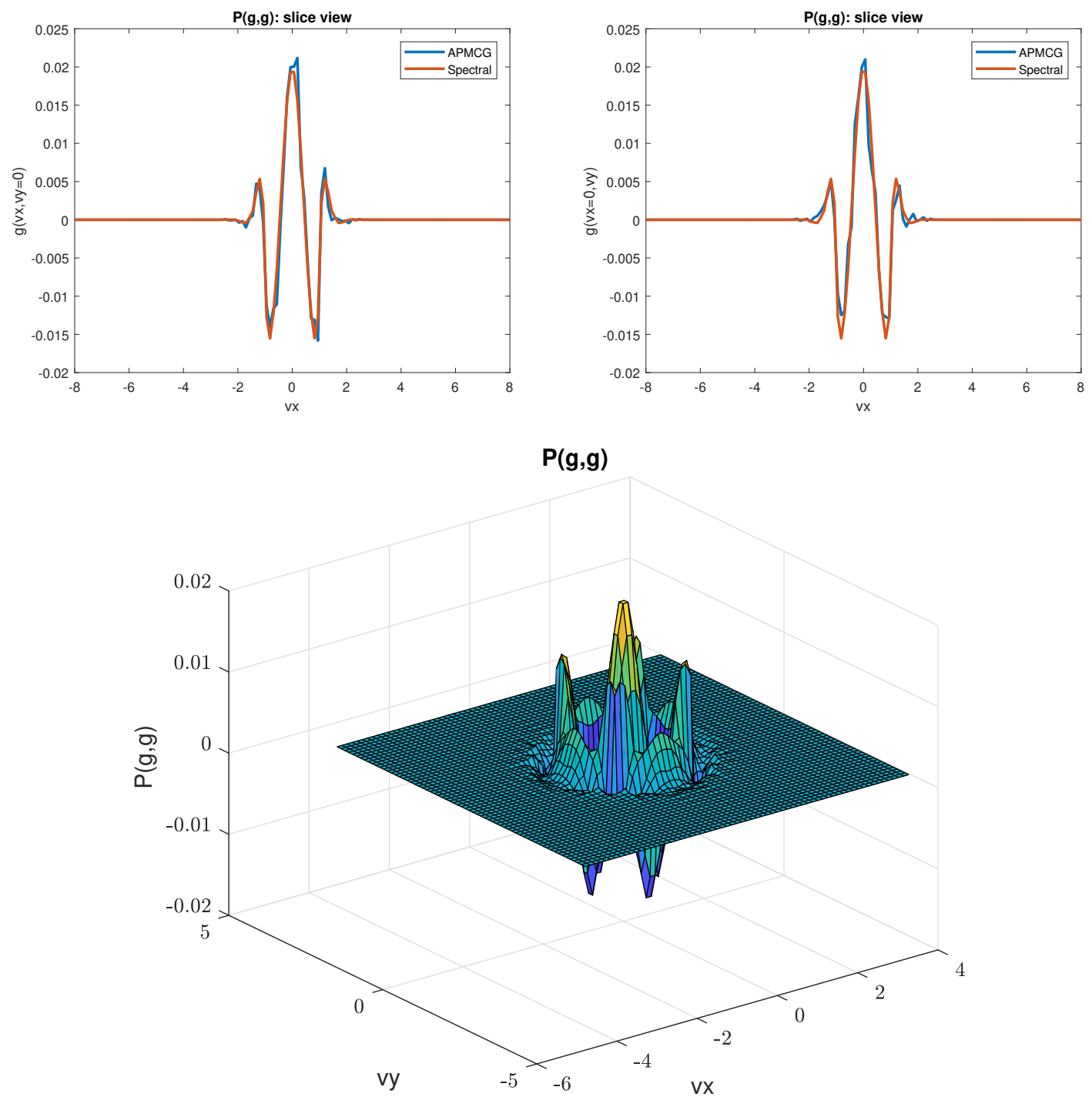

Figure 4: Computation of $P(g, g)(v)$ by the APMCG and by the spectral methods. Top left: one-dimensional slice $P(g, g)\left(v_{x}, 0\right)$. Top right: one-dimensional slice $P(g, g)\left(0, v_{y}\right)$. Bottom: two-dimensional $P(g, g)(v)$ using APMCG.

\subsection{Analysis of the full numerical scheme}

In this part, we study the dynamics of the solution to the homogeneous Boltzmann equation (10). To do so, the initial data (52) is considered and we use $N=10^{6}$ to sample the initial distribution $f(v, t=0)$. The time step is $\Delta t=0.2$ and the final time is $T=5$. As previously, we also consider a reference solution computed by the spectral method with the same velocity numerical parameters as before and with $\Delta t=0.2$.

In Figure 12, we show the two-dimensional distribution function at different times $(t=$ 

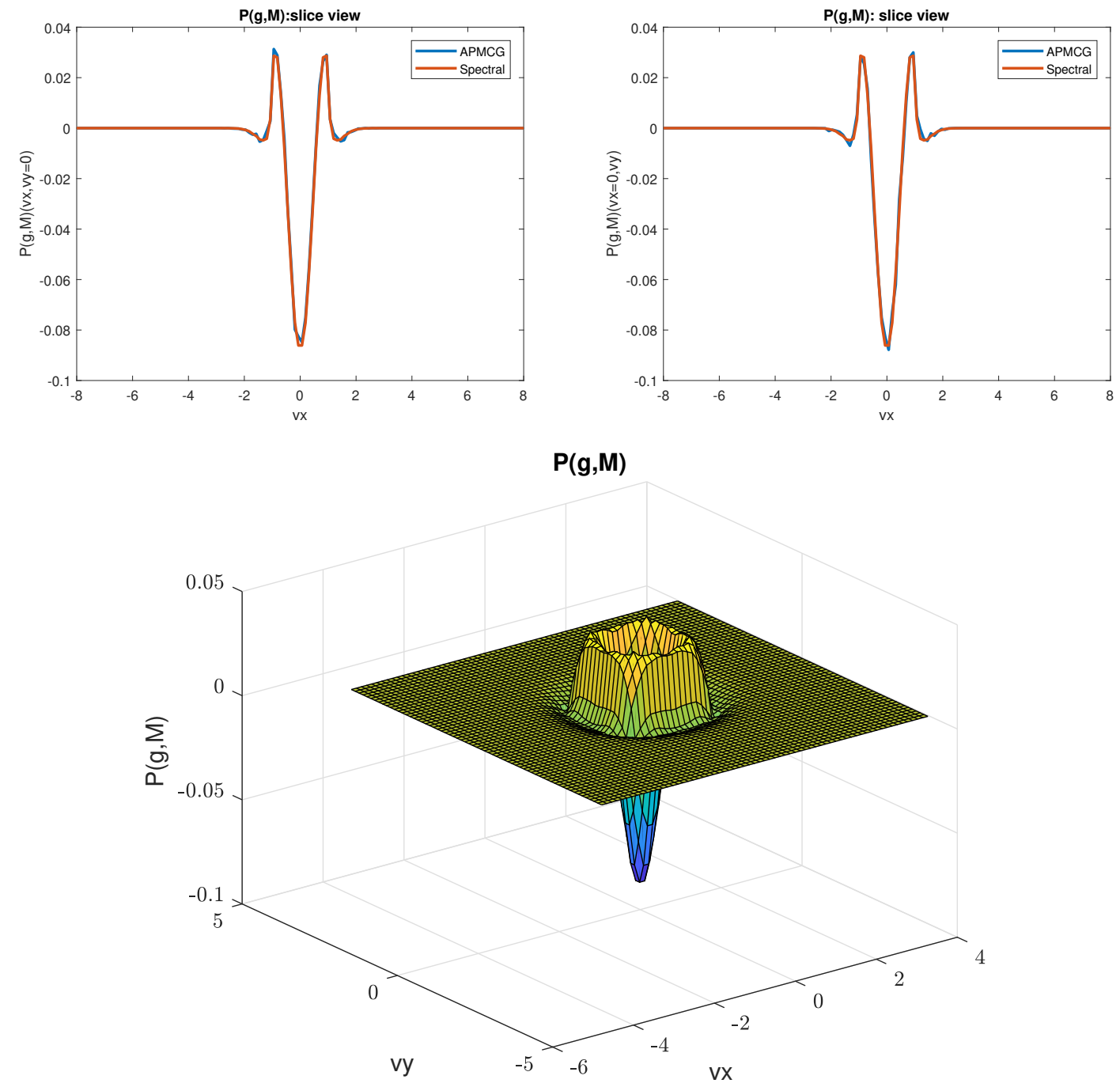

Figure 5: Computation of $P(g, M)(v)$ by the APMCG and by the spectral methods. Top left: one-dimensional slice $P(g, M)\left(v_{x}, 0\right)$. Top right: one-dimensional slice $P(g, M)\left(0, v_{y}\right)$. Bottom: two-dimensional $P(g, M)(v)$ using APMCG.

$0.4,1.2,3.6)$ as well as the two-dimensional pointwise error between the APMCG and the spectral solutions. One can observe that the error remains small (about $10^{-3}$ ) which confirms the good behavior of APMCG. Next, in Figure 13 (left), we show the time evolution of the error (in $L^{1}$ norm) for both the standard Monte Carlo and APMCG methods (the reference solution is computed using the spectral method). The error for the two methods is quite similar (even slightly better for APMCG) but if we look at the time evolution of the number of particles in Figure 13 (right), we observe that for the new APMCG method, it decreases very quickly (with an exponentially rate) whereas it remains constant for the standard MC method. More than that, the error becomes smaller when the equilibrium is approached which emphasizes 

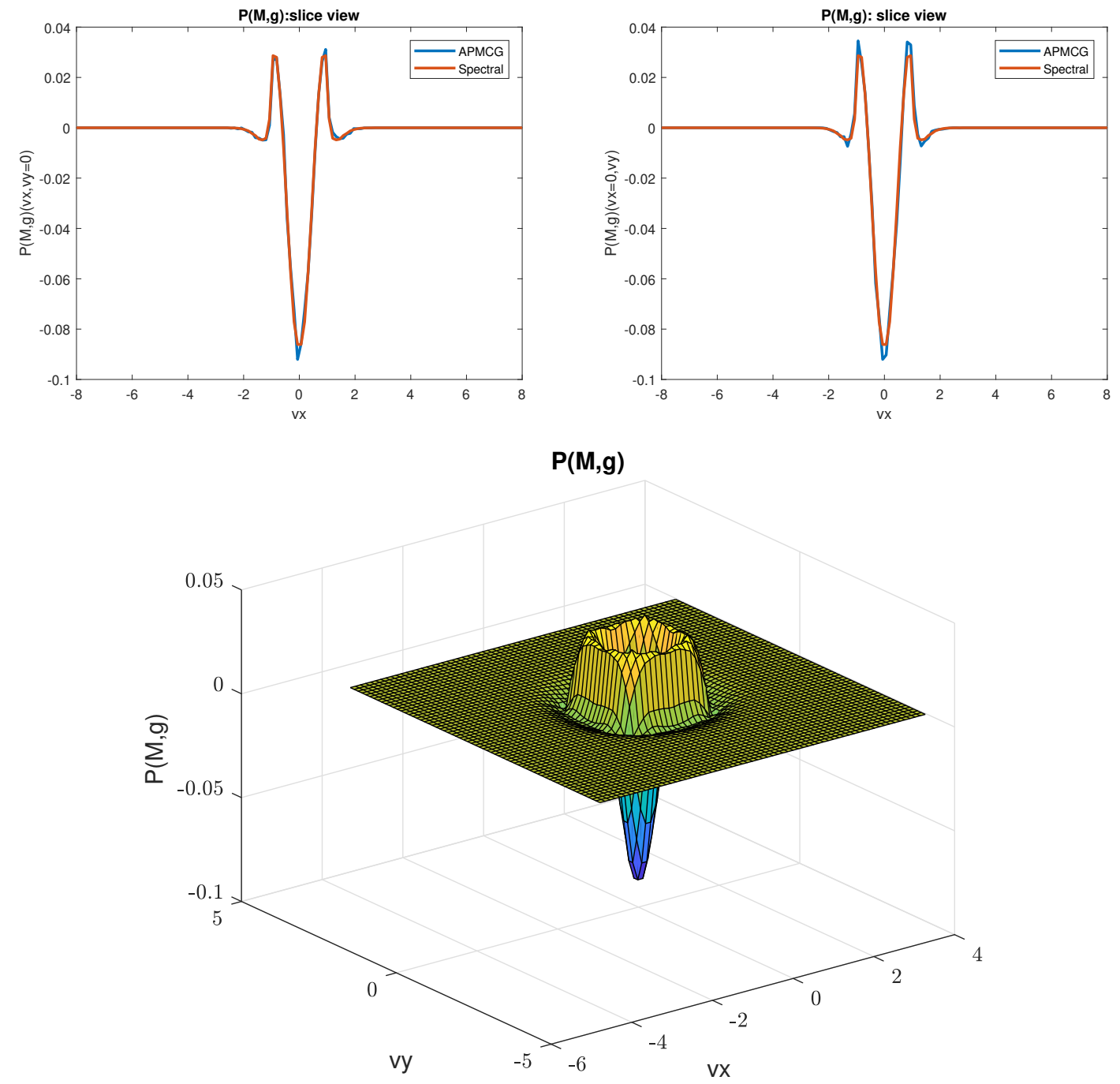

Figure 6: Computation of $P(M, g)(v)$ by the APMCG and by the spectral methods. Top left: one-dimensional slice $P(M, g)\left(v_{x}, 0\right)$. Top right: one-dimensional slice $P(M, g)\left(0, v_{y}\right)$. Bottom: two-dimensional $P(M, g)(v)$ using APMCG.

the strength of the method in this regime. Indeed, the cost of the new method, which can be measured by the number of samples present in the computational domain, diminishes with time. At the beginning we employ almost half of the number of particles used by the standard MC and we end with less than $10 \%$ of the initial number $N$ while the MC maintains a constant number during all the simulation. 

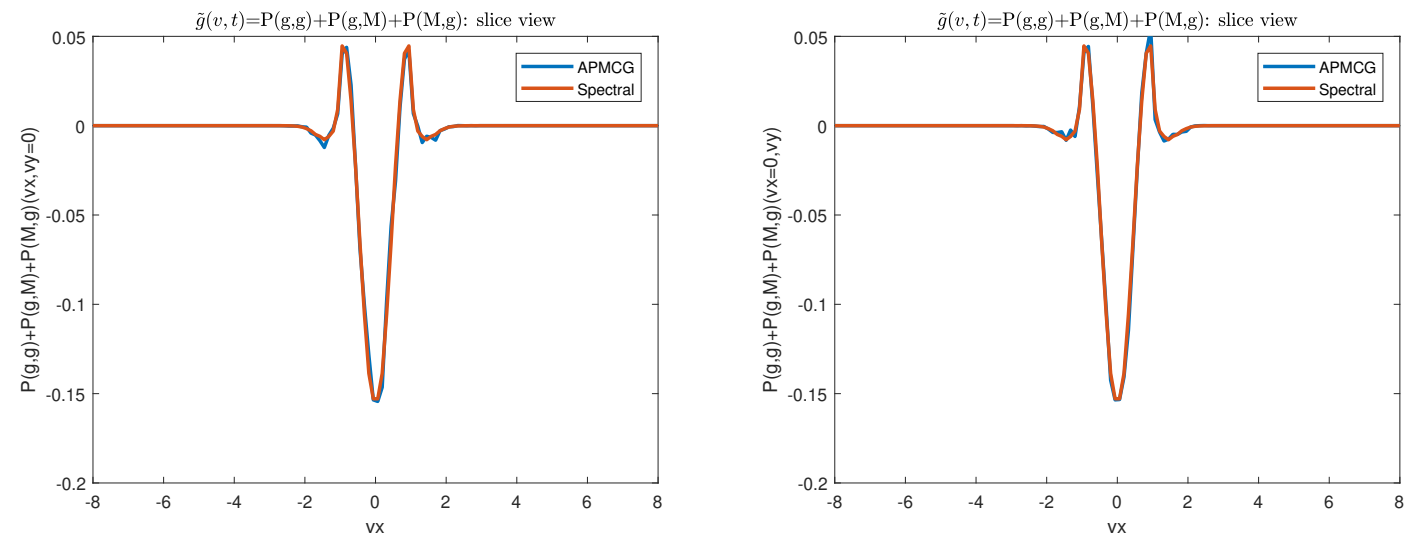

Figure 7: Slices of the sum of the three collisional operators $\tilde{g}(v)$ using the spectral and the APMCG methods. Left: $\tilde{g}\left(v_{x}, 0\right)$. Right: $\tilde{g}\left(0, v_{y}\right)$.

$\tilde{g}(v, t)=\mathrm{P}(\mathrm{g}, \mathrm{g})+\mathrm{P}(\mathrm{g}, \mathrm{M})+\mathrm{P}(\mathrm{M}, \mathrm{g})$ Spectral

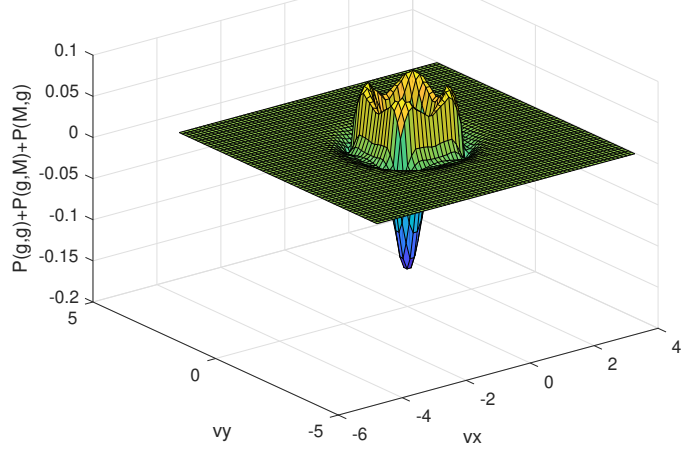

$\tilde{g}(v, t)=\mathrm{P}(\mathrm{g}, \mathrm{g})+\mathrm{P}(\mathrm{g}, \mathrm{M})+\mathrm{P}(\mathrm{M}, \mathrm{g})$ APMCG

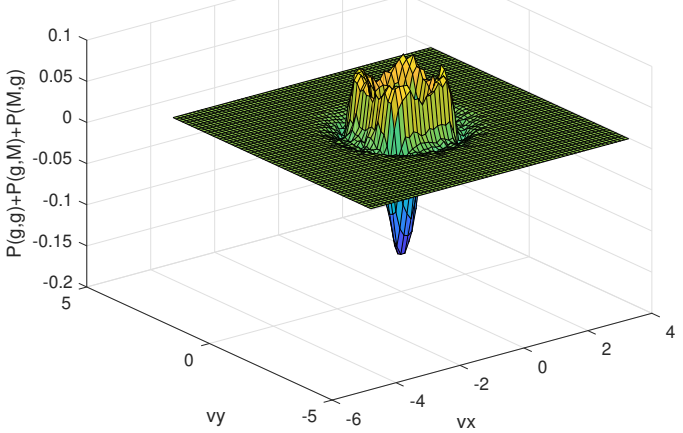

Pointwise Error

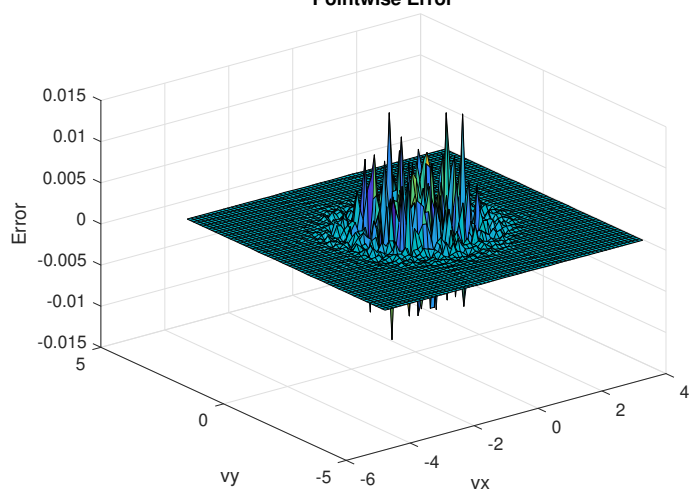

Figure 8: Two-dimensional representation of the sum of the three collisional operators $\tilde{g}(v)$ using the spectral and the APMCG methods. Top left: spectral method. Top right: APMCG method. Bottom: pointwise error between the spectral and the APMCG methods. 

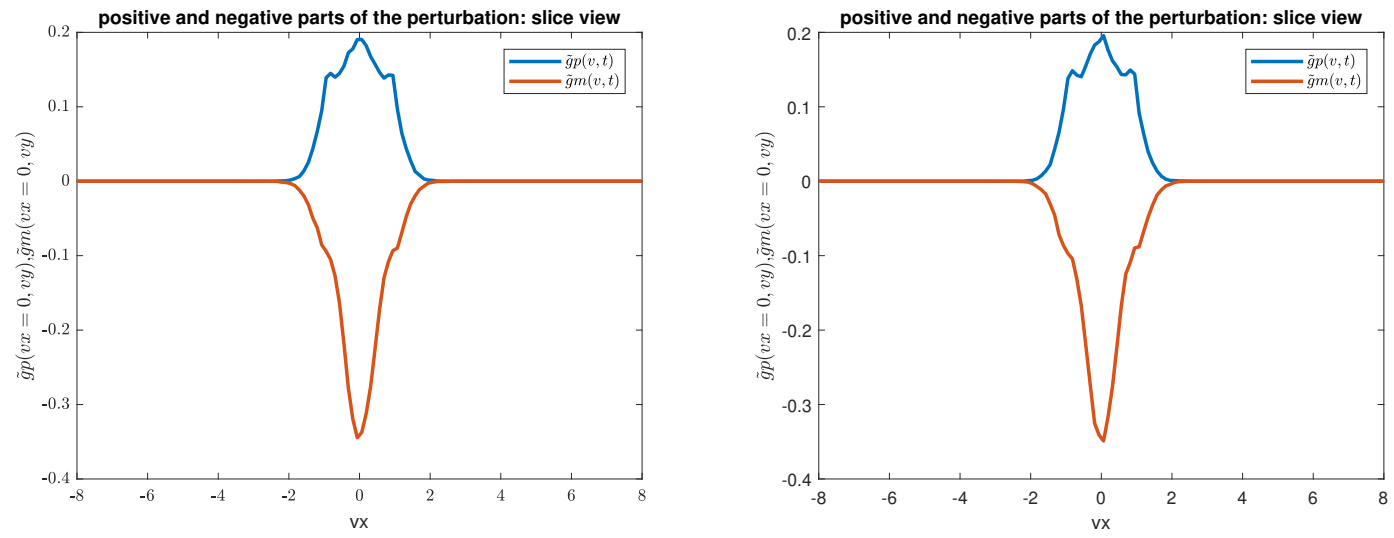

positive and negative parts of the perturbation: $\tilde{g} p(v x, v y), \tilde{g} m(v x, v y)$

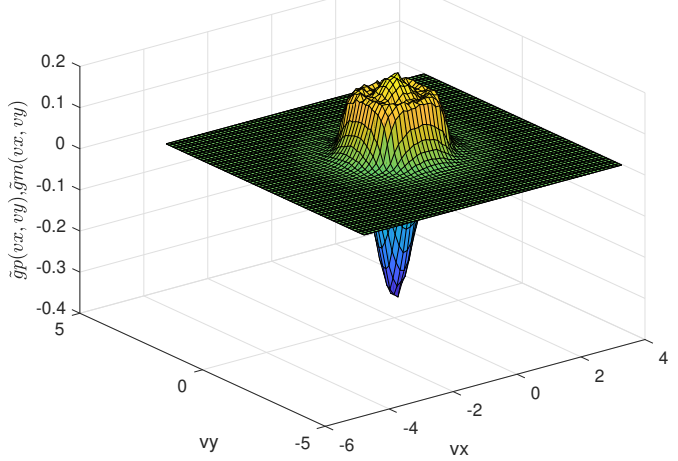

Figure 9: Representation of the sum of the three collisional operators $\tilde{g}_{p / m}(v)$ (positive and negative part). Top left: slice views of $\tilde{g}_{p / m}\left(v_{x}, 0\right)$. Top right: $\tilde{g}_{p / m}\left(0, v_{y}\right)$. Bottom: $\tilde{g}(v)$.

\section{Conclusions}

In this work, we have presented a new Monte Carlo method for the numerical approximation of the solution to the Boltzmann equation. This new method enjoys the following properties: (i) its statistical noise is smaller than the one of standard Monte Carlo methods; (ii) it is asymptotically stable with respect to the Knudsen number; (iii) its computational cost as well as its variance diminish as the equilibrium is approached. The method is based on a micromacro decomposition and on an exponential Runge-Kutta method for the time discretization. The Monte Carlo method is constructed to describe the deviation from the thermodynamical equilibrium. This enables to derive a low variance Monte Carlo method for which the number of particles used to sample the unknown diminishes automatically as the equilibrium state is approached. The numerical results illustrate the efficiency of the proposed method compared to the standard Monte Carlo approach and its accuracy compared to the spectral method.

A natural extension of this work would be the case of non homogeneous Boltzmann equation. In this configuration, the micro-macro decomposition leads to a coupled fluid/kinetic model for which a hybrid deterministic-MC (deterministic for the equilibrium part and MC for the deviational part) method needs to be developed. 


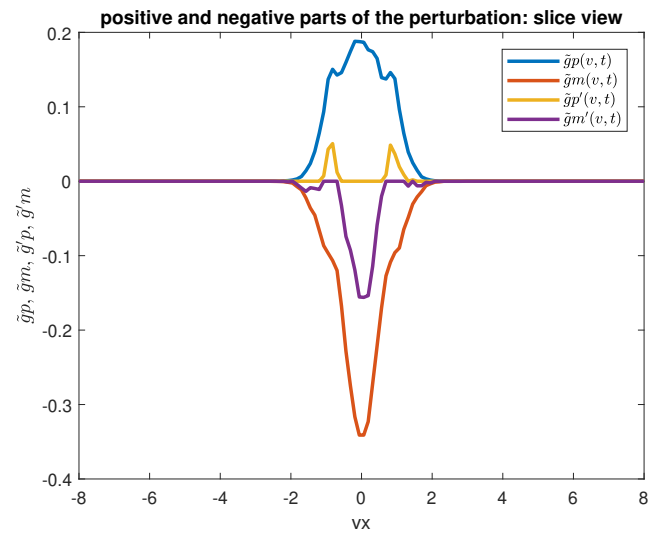

positive part of the perturbation: $\tilde{g}^{\prime} p(v x, v y)$

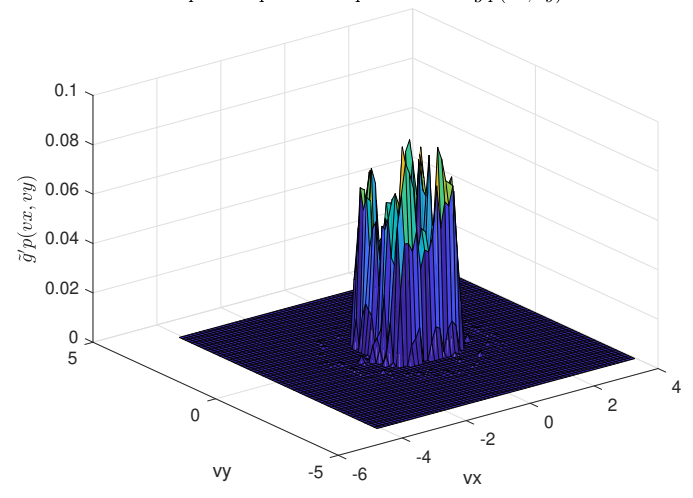

perturbation: $\tilde{g}^{\prime}(v x, v y)$

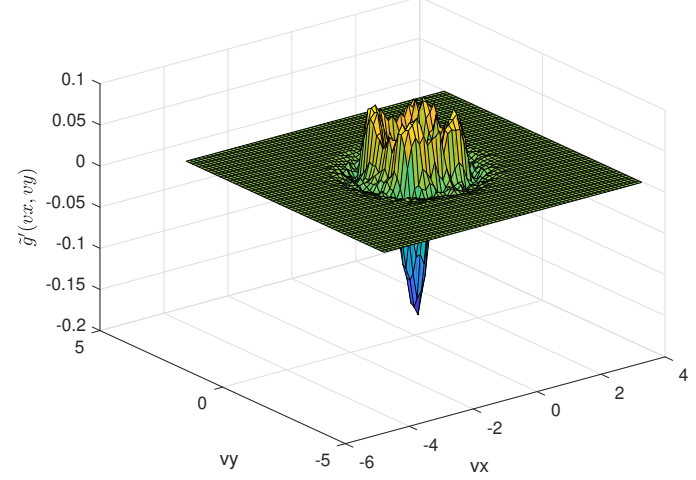

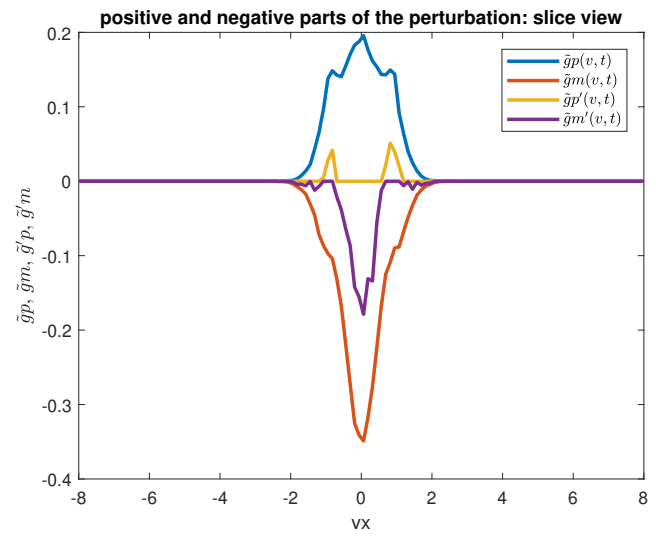

negative part of the perturbation: $\tilde{g}^{\prime} m(v x, v y)$

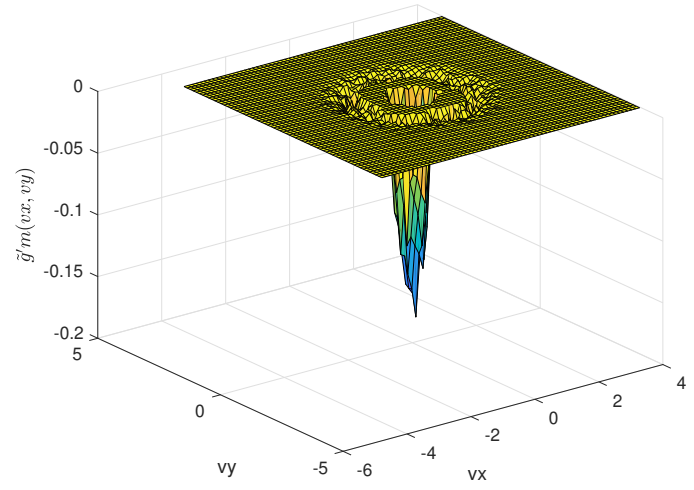

Pointwise Error for $\tilde{g}^{\prime}(v x, v y)$

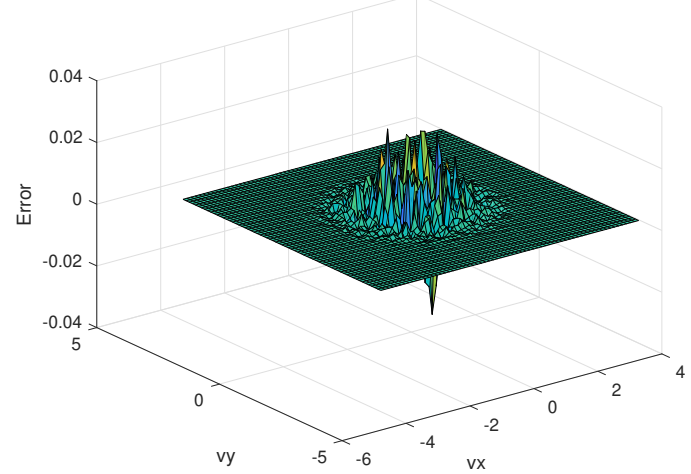

Figure 10: Representation of the sum of the three collisional operators $\tilde{g}_{p / m}(v)$ together with the KDE approximation $\hat{g}_{p / m}(v)$ (positive and negative part). Top left: slice views of $\tilde{g}_{p / m}\left(v_{x}, 0\right)$ and $\hat{g}_{p / m}\left(v_{x}, 0\right)$. Top right: $\tilde{g}_{p / m}\left(0, v_{y}\right)$ and $\hat{g}_{p / m}\left(0, v_{y}\right)$. Middle left: $\hat{g}_{p}(v)$. Middle right: $\hat{g}_{p}(v)$. Bottom left: $\hat{g}(v)$. Bottom right: pointwise error between APMCG and the spectral method.

\section{A A Fast Spectral Scheme for the Boltzmann collision operator}

The fast spectral discretization of the Boltzmann operator employed for testing our Monte Carlo method is described in this Appendix. For details on this method, we refer to [29, 49]. Since 


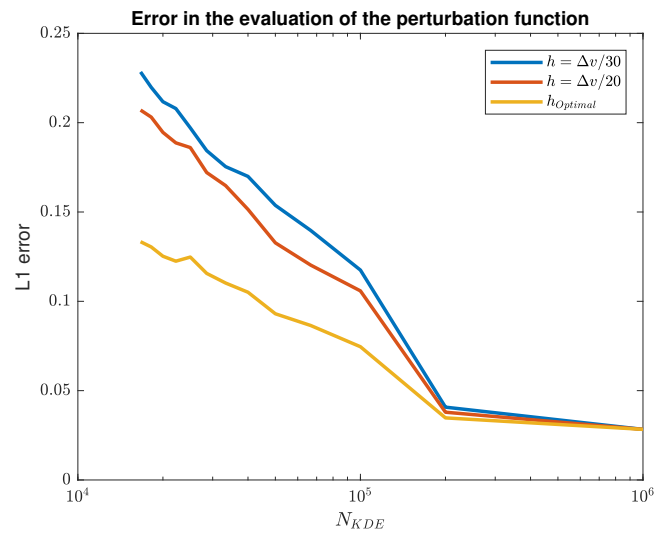

Figure 11: Error ( $L^{1}$ norm) between APMCG (for different values $N_{K D E}$ and for different values of the bandwidth $h$ ) and the spectral method. $\Delta v=16 / 128$ denotes the mesh size used for the spectral method.

the collision operator acts only on the velocity variable, only this dependency is considered, i.e. $f=f(v)$. Using one index to denote the $d_{v}$-dimensional sums, we have that the approximate function $f_{N_{v}}$ can be represented as the truncated Fourier series by

$$
f_{N_{v}}(v)=\sum_{|k| \leq N_{v} / 2} \hat{f}_{k} e^{i k \cdot v}
$$

with $k=\left(k_{1}, \cdots, k_{d_{v}}\right) \in \mathbb{N}^{d_{v}},|k|=\max \left\{\left|k_{i}\right|, 1 \leq i \leq d_{v}\right\}$ and where the Fourier coefficients are given by

$$
\hat{f}_{k}=\frac{1}{(2 \pi)^{d_{v}}} \int_{[-\pi, \pi]^{d v}} f(v) e^{-i k \cdot v} d v
$$

The first step of the method consists in a truncation of the integration domain of the Boltzmann integral (2). As a consequence, the distribution function has compact support on the ball $\mathcal{B}_{0}(R)$ of radius $R$ centred in the origin. To avoid aliasing issues, typical of spectral approximations, it is sufficient that the support of $f(v)$ is restricted to the cube $[-T, T]^{d v}$ with $T \geq(2+\sqrt{2}) R$, since $\operatorname{supp}(Q(f)(v)) \subset \mathcal{B}_{0}(\sqrt{2} R)$. Successively, one fixes $f(v)=0$ on $[-T, T]^{d_{v}} \backslash \mathcal{B}_{0}(R)$ and extended it to a periodic function on the set $[-T, T]^{d_{v}}$. In the following, we do the choice $T=(3+\sqrt{2}) R / 2$, we fix $T=\pi$ and hence $R=\lambda \pi$ with $\lambda=2 /(3+\sqrt{2})$. By denoting with $Q^{R}(f, f)$ the Boltzmann operator with cut-off, we obtain a spectral quadrature of the operator by projecting (2) on the space of trigonometric polynomials of degree less or equal to $N_{v}$, i.e.

$$
\hat{Q}_{k}=\int_{[-\pi, \pi]^{d v}} Q^{R}\left(f_{N_{v}}, f_{N_{v}}\right) e^{-i k \cdot v} d v, \quad|k| \leq N_{v} / 2,
$$

and by substituting expression (54) in (56) one gets after some computations

$$
\hat{Q}_{k}=\sum_{\substack{|\ell|,|m| \leq N / 2 \\ \ell+m=k}} \hat{f}_{\ell} \hat{f}_{m} \hat{\beta}(\ell, m), \quad|k| \leq N_{v} / 2,
$$


Distribution function at time 0.4. APMCG

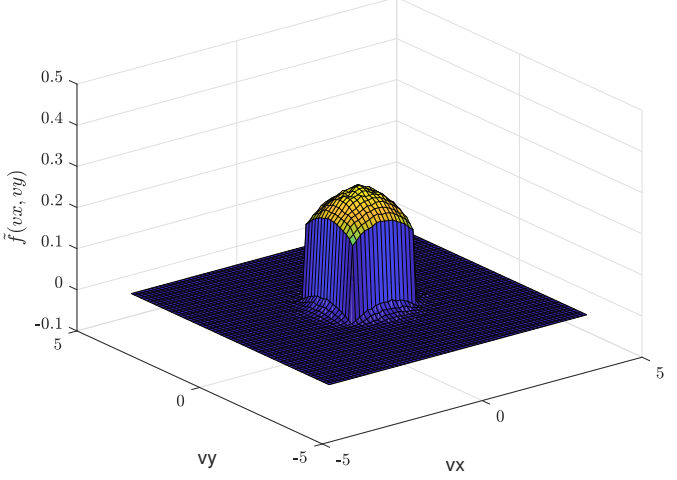

Distribution function at time 1.2. APMCG

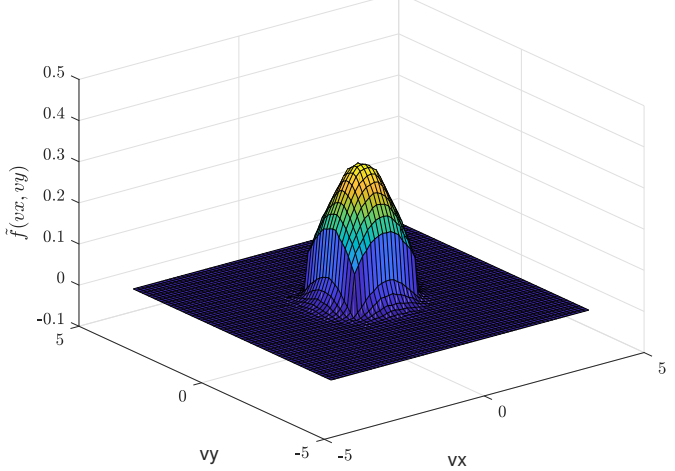

Distribution function at time 3.6. APMCG

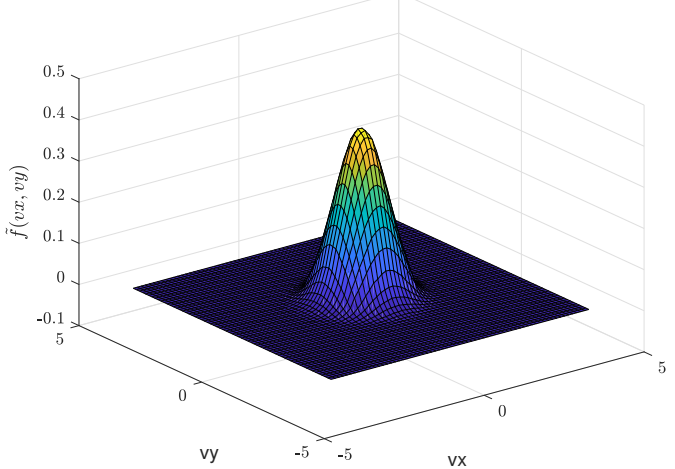

Pointwise Error for $g(v x, v y)$ at time 0.4. APMCG

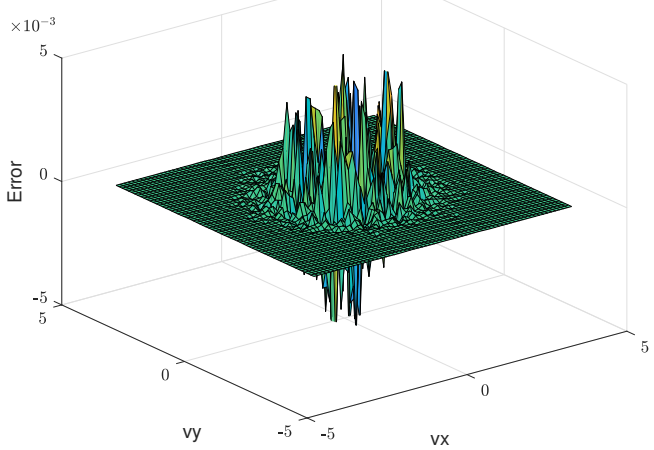

Pointwise Error for $g(v x, v y)$ at time 1.2. APMCG

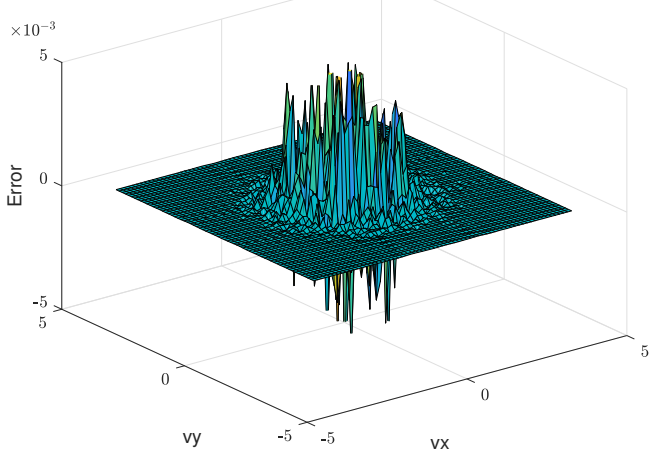

Pointwise Error for $g(v x, v y)$ at time 3.6. APMCG

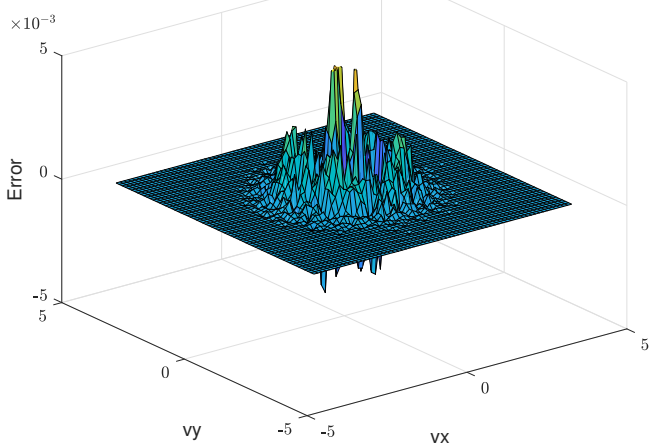

Figure 12: Left column: Two-dimensional distribution function $f(v, t)$ at different times $t=0.4,1.2,3.6$. Right column: two-dimensional pointwise error between $f(v, t)$ (at times $t=0.4,1.2,3.6)$ and the reference solution obtained by the spectral method.

where $\hat{\beta}(\ell, m)=\hat{B}(\ell, m)-\hat{B}(m, m)$ are given by

$$
\hat{B}(\ell, m)=\int_{\mathcal{B}_{0}(2 \lambda \pi)} \int_{\mathbb{S}^{d v}-1}|q| \sigma(|q|, \cos \theta) e^{-i\left(\ell \cdot q^{+}+m \cdot q^{-}\right)} d \omega d q .
$$



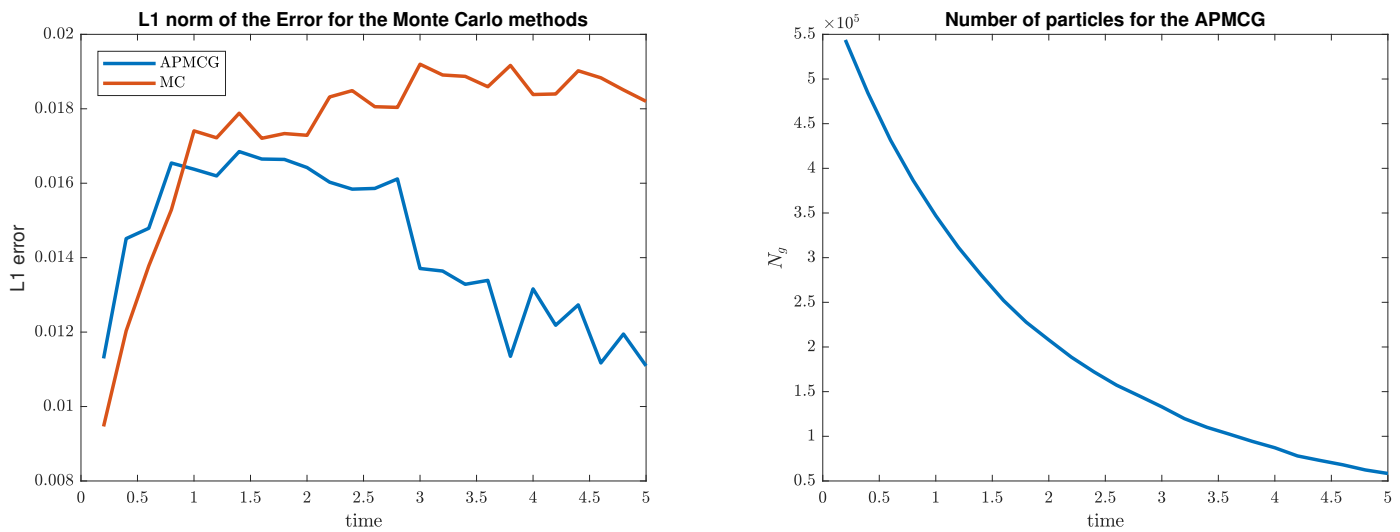

Figure 13: Right: error ( $L^{1}$ norm) for the Monte Carlo and APMCG as a function of time (the reference solution is obtained with the spectral method). Left: time evolution of the number of particles for APMCG.

with

$$
q^{+}=\frac{1}{2}(q+|q| \omega), \quad q^{-}=\frac{1}{2}(q-|q| \omega) .
$$

This is enough to define a possible spectral discretization of the Boltzmann operator. However, let notice that the naive evaluation of (57) requires $O\left(n^{2}\right)$ operations, where $n=N_{v}^{d_{v}}$. This causes the spectral method to be computationally very expensive. Thus, in order to reduce the number of operations needed to evaluate the collision integral, the main idea is to use another representation of (2), the so-called Carleman representation which reads

$$
Q(f, f)(v)=\int_{\mathbb{R}^{d_{v}}} \int_{\mathbb{R}^{d_{v}}} \tilde{B}(x, y) \delta(x \cdot y)[f(v+y) f(v+x)-f(v+x+y) f(v)] d x d y,
$$

with

$$
\tilde{B}(|x|,|y|)=2^{d_{v}-1} \sigma\left(\sqrt{|x|^{2}+|y|^{2}}, \frac{|x|}{\sqrt{|x|^{2}+|y|^{2}}}\right)\left(|x|^{2}+|y|^{2}\right)^{-\frac{d_{v}-2}{2}} .
$$

This transformation yields the following new spectral quadrature formula

$$
\hat{Q}_{k}=\sum_{\substack{|\ell|,|m| \leq N_{v} / 2 \\ \ell+m=k}} \hat{\beta}_{F}(\ell, m) \hat{f}_{\ell} \hat{f}_{m}, \quad|k| \leq N_{v} / 2,
$$

where $\hat{\beta}_{F}(\ell, m)=\hat{B}_{F}(\ell, m)-\hat{B}_{F}(m, m)$ are now given by

$$
\hat{B}_{F}(\ell, m)=\int_{\mathcal{B}_{0}(R)} \int_{\mathcal{B}_{0}(R)} \tilde{B}(x, y) \delta(x \cdot y) e^{i(\ell \cdot x+m \cdot y)} d x d y .
$$

The main difference is that now equation (62) can be recast in a convolutive form. This can be done if one is able to write $\hat{\beta}_{F}(\ell, m)$ as

$$
\hat{\beta}_{F}(\ell, m) \simeq \sum_{p=1}^{A} \alpha_{p}(\ell) \alpha_{p}^{\prime}(m),
$$


where $A$ represents the number of finite possible angles which defines the directions of collisions. This permits to evaluate the collision integral as a sum of $A$ discrete convolutions. This can be done at a cost $O\left(A N_{v}^{d_{v}} \log _{2} N_{v}^{d_{v}}\right)$ by means of standard FFT techniques. In fact, one can notice that for Maxwellian molecules in dimension $d_{v}=2$, i.e. the case considered in the simulations, one has from (61) that $\tilde{B}$ is constant. Thus, by rewriting $x$ and $y$ in spherical coordinates $x=\rho e$ and $y=\rho^{\prime} e^{\prime}$, one ends with

$$
\hat{B}_{F}(\ell, m)=\frac{1}{4} \int_{\mathbb{S}^{1}} \int_{\mathbb{S}^{1}} \delta\left(e \cdot e^{\prime}\right)\left[\int_{-R}^{R} e^{i \rho(\ell \cdot e)} d \rho\right]\left[\int_{-R}^{R} e^{i \rho^{\prime}\left(m \cdot e^{\prime}\right)} d \rho^{\prime}\right] d e d e^{\prime} .
$$

Denoting $\phi_{R}^{2}(s)=\int_{-R}^{R} e^{i \rho s} d \rho$, for $s \in \mathbb{R}$, we have the explicit formula

$$
\phi_{R}^{2}(s)=2 R \operatorname{Sinc}(R s), \quad \operatorname{Sinc}(x)=\frac{\sin (x)}{x} .
$$

This formula is further plugged in the expression of $\hat{B}_{F}(\ell, m)$ yielding

$$
\hat{B}_{F}(\ell, m)=\int_{0}^{\pi} \phi_{R}^{2}\left(\ell \cdot e_{\theta}\right) \phi_{R}^{2}\left(m \cdot e_{\theta+\pi / 2}\right) d \theta .
$$

Finally, a regular discretization of $A$ in equally spaced points gives

$$
\hat{B}_{F}(\ell, m)=\frac{\pi}{M} \sum_{p=1}^{A} \alpha_{p}(\ell) \alpha_{p}^{\prime}(m),
$$

with

$$
\alpha_{p}(\ell)=\phi_{R}^{2}\left(\ell \cdot e_{\theta_{p}}\right), \quad \alpha_{p}^{\prime}(m)=\phi_{R}^{2}\left(m \cdot e_{\theta_{p}+\pi / 2}\right)
$$

where $\theta_{p}=\pi p / A, p=1, \ldots, A$. In the numerical simulations shown, the number of discretization angles has been fixed to $A=8$.

\section{A New algorithm}

In this Appendix, an alternative algorithm (to Algorithm 5.2) is presented which still enjoys the AP property and which also makes the number of particles decrease when the unknown $f$ becomes close to the Maxwellian equilibrium. The main goal is to control the number of particles that are created through the sampling of the collision operators. A possible way to decrease the increasing factor function $I F(\mu \Delta t / \varepsilon$ ) (defined in (45)) consists in recasting the APMCG method by rewriting the collisional part of equation (37) summing up one part:

$$
P\left(g_{p}^{n}, g_{p}^{n}\right)+P\left(g_{p}^{n}, g_{m}^{n}\right)+P\left(g_{m}^{n}, g_{m}^{n}\right)+P\left(g_{m}^{n}, g_{p}^{n}\right)+P\left(g_{p}^{n}, M\right)+P\left(g_{m}^{n}, M\right)=P\left(g^{n}, f^{n}\right) .
$$

This permits to reduce the Increasing Factor discarding the term $\mu_{g} / \mu$ :

$$
I F(\mu \Delta t / \varepsilon)=\left(e^{-\frac{\mu \Delta t}{\varepsilon}}+2 \frac{\mu \Delta t}{\varepsilon} e^{-\frac{\mu \Delta t}{\varepsilon}}\right) \leq 1
$$

The new algorithm reads: 
Algorithm A.1. Asymptotic Preserving Monte Carlo (APMCG2) method for Maxwell molecules.

- Compute the initial velocities of the particles belonging to the approximation of $g_{p}(v, t=0)$ and $g_{m}(v, t=0):\left\{v_{p, 1}(t=0), . ., v_{p, N_{p}}(t=0)\right\},\left\{v_{m, 1}(t=0), . ., v_{m, N_{m}}(t=0)\right\}$.

- from $n=1$ to $n=n_{\text {fin }}$

- set $N K_{p}=\operatorname{Iround}\left(e^{-\frac{\mu \Delta t}{\varepsilon}} N_{p}(t)\right)$ and select $N K_{p}$ particles uniformly. Denote $I_{p}$ the corresponding subset of indices. Set $v_{p, k}^{n+1}=v_{p, k}^{n}$ for $k \in I_{p}$.

- set $N K_{m}=\operatorname{Iround}\left(e^{-\frac{\mu \Delta t}{\varepsilon}} N_{m}(t)\right)$ and select $N K_{m}$ particles uniformly. Denote $I_{m}$ the corresponding subset of indices. Set $v_{m, k}^{n+1}=v_{m, k}^{n}$ for $k \in I_{m}$.

- discard a random fraction $N D_{p}=\operatorname{Iround}\left(\left(1-e^{-\frac{\mu \Delta t}{\varepsilon}}-\frac{\mu \Delta t}{\varepsilon} e^{-\frac{\mu \Delta t}{\varepsilon}}\right) N_{p}(t)\right)$.

- discard a random fraction $N D_{m}=\operatorname{Iround}\left(\left(1-e^{-\frac{\mu \Delta t}{\varepsilon}}-\frac{\mu \Delta t}{\varepsilon} e^{-\frac{\mu \Delta t}{\varepsilon}}\right) N_{m}(t)\right)$.

- sampling of $P(g, f)$ : keep a fraction $N C_{1}=\operatorname{Iround}\left(\frac{\mu \Delta t}{\varepsilon} e^{-\frac{\mu \Delta t}{\varepsilon}}\left(N_{p}(t)+N_{m}(t)\right)\right)$. Denote $I_{c 1}$ the random set (of size $N C_{1}$ ) of particles indices and $S c 1=\left\{v_{k}^{n}\right\}_{k \in I_{c 1}}$ composed of positive or negative particles. Denote $S c 2=S c 1 \cup\left\{v_{M, k}\right\}_{k=1, \ldots, N C}$ with $v_{M, k}$ are the particles sampled from the Maxwellian distribution $M$.

a) compute $v_{k}^{\prime}$ by performing a collision between particle $k$ whose velocity $v_{k} \in S c 1$ and a random particle $j$ whose velocity $v_{j} \in S c 2$.

b) assign $v_{p, k}^{n+1}=v_{k}^{\prime} \quad$ if both particles belong to the same category (positive or negative) or if one belongs to the positive category and the second is Maxwellian, $v_{m, k}^{n+1}=v_{k}^{\prime} \quad$ if the particles do not belong to the same category or if one belongs to the negative category and the second is Maxwellian.

- sampling of $P(M, g)$ : keep a fraction $N C_{2}=\operatorname{Iround}\left(\frac{\mu \Delta t}{\varepsilon} e^{-\frac{\mu \Delta t}{\varepsilon}}\left(N_{p}(t)+N_{m}(t)\right)\right)$. Denote $I_{c 2}$ the random set (of size $N C_{2}$ ) of particles indices and $S c 2=\left\{v_{k}^{n}\right\}_{k \in I_{c 2}}$ composed of positive or negative particles.

a) compute $v_{k}^{\prime}$ by performing a collision between the particle with velocity $v_{M, k}$ and a random particle taken from the set $S_{c 2}$.

b) assign $v_{p, k}^{n+1}=v_{k}^{\prime} \quad$ if the random particle $k$ belonged to the positive category, $v_{m, k}^{n+1}=v_{k}^{\prime} \quad$ if the random particle $k$ belonged to the negative category.

- end loop over time

\section{Acknowledgments}

A. Crestetto is supported by the French ANR projects MoHyCon ANR-17-CE40-0027-01.

\section{References}

[1] H. Babovsky, On a simulation scheme for the Boltzmann equation, Math. Methods Appl. Sci., 8 (1986), pp. 223-233. 
[2] C. Bardos, F. Golse, D. Levermore, Fluid dynamics limits of kinetic equations I. Formal derivation, J. Stat. Phys., 63 (1991), pp. 323-344.

[3] M. Bennoune, M. Lemou, L. Mieussens, Uniformly stable numerical schemes for the Boltzmann equation preserving the compressible Navier-Stokes asymptotics, J. Comput. Phys., 227 (2008), pp. 3781-3803.

[4] G. A. BIRD, Molecular gas dynamics and direct simulation of gas flows, Clarendon Press, Oxford 1994.

[5] A. V. Bobylev, J. Struckmeier, Numerical simulation of the stationary onedimensional Boltzmann equation by particle methods, European J. Mech. B Fluids, 15 (1996), pp. 103-118.

[6] Z. I. Botev, J. F. Grotowski, D. P. Kroese, Kernel density estimation via diffusion, Annals of Statistics, 38 (2010), pp. 2916-2957.

[7] S. Brunner, E. Valeo, J. A. Krommes, Collisional delta-f scheme with evolving background for transport time scale simulations, Phys. of Plasmas, 12 (1999).

[8] S. Brunner, E. Valeo, J. A. Krommes, Linear delta-f simulations of nonlocal electron heat transport, Phys. of Plasmas, 7 (2000).

[9] J. Burt, I. Boyd, A hybrid particle approach for continuum and rarefied flow simulation, J. Comput. Phys., 228 (2009), pp. 460-475.

[10] R. Caflisch, Monte Carlo and Quasi-Monte Carlo Methods, Acta Numerica, 7 (1998), pp. $1-49$.

[11] R. Caflisch, G. Dimarco, L. Pareschi, Direct simulation Monte Carlo schemes for Coulomb interactions in plasmas, Commun. Appl. Ind. Math., 1 (2010), pp. 72-91.

[12] R. Caflisch, C. Wang, G. Dimarco, B. Cohen, A. Dimits, A hybrid method for accelerated simulation of Coulomb collisions in a plasma, SIAM MMS, 7 (2008), pp. 865887.

[13] M. Campos Pinto, E. Sonnendrücker, A. Friedman, D. Grote, S. Lund, Noiseless Vlasov-Poisson simulations with linearly transformed particles, J. Comput. Phys., 275 (2014), pp. 236-256.

[14] M. Campos Pinto, Towards smooth particle methods without smoothing, J. Sci. Comput., 65 (2015), pp. 54-82.

[15] M. Campos Pinto, F. Charles, Uniform convergence of a linearly transformed particle method for the Vlasov-Poisson system, SIAM J. Numer. Anal., 54 (2016), pp. 137-160.

[16] C. Cercignani, The Boltzmann equation and its applications, Springer-Verlag, New York, 1988. 
[17] A. Crestetto, N. Crouseilles, M. Lemou, Kinetic/fluid micro-macro numerical schemes for Vlasov-Poisson-BGK equation using particles, Kin. Rel. Models, 5 (2012), pp. 787-816.

[18] A. Crestetto, N. Crouseilles, G. Dimarco, M. Lemou, Asymptotically complexity diminishing schemes (ACDS) for kinetic equations in the diffusive scaling, J. Comput. Phys., 394 (2019), pp. 243-262.

[19] N. Crouseilles, G. Dimarco, M. Lemou, Asymptotic preserving and time diminishing schemes for rarefied gas dynamics, Kin. Rel. Models, 10 (2017), pp. 643-668.

[20] N. Crouseilles, M. Lemou, An asymptotic preserving scheme based on a micro-macro decomposition for collisional Vlasov equations: diffusion and high-field scaling limits, Kin. Rel. Models, 4 (2011), pp. 441-477.

[21] P. Degond, G. Dimarco, L. Pareschi, The moment guided Monte Carlo method, International Journal for Numerical Methods in Fluids, 67 (2011), pp. 189-213.

[22] P. Degond, G. Dimarco, Fluid simulations with localized Boltzmann upscaling by direct simulation Monte-Carlo, J. Comput. Phys., 231 (2012), pp. 2414-2437.

[23] G. Dimarco, The hybrid moment guided Monte Carlo method for the Boltzmann equation, Kin. Rel. Models, 6 (2013), pp. 291-315.

[24] G. Dimarco, L. Pareschi, Hybrid multiscale methods II. Kinetic equations, SIAM MMS, 6 (2007), pp. 1169-1197.

[25] G. Dimarco, L. Pareschi, A fluid solver independent hybrid method for multiscale kinetic equations, SIAM J. Sci. Comput., 32 (2010), pp. 603-634.

[26] G. Dimarco, L. Pareschi, Exponential Runge-Kutta methods for stiff kinetic equations, SIAM J. Numer. Anal., 49 (2011), pp. 2057-2077.

[27] G. Dimarco, L. Pareschi, High order asymptotic-preserving schemes for the Boltzmann equation, C. R. Math. Acad. Sci. Paris, 350 (2012), pp. 481-486.

[28] G. Dimarco, L. Pareschi, Asymptotic preserving implicit-explicit Runge-Kutta methods for non linear kinetic equations, SIAM J. Num. Anal., 32 (2013), pp. 1064-1087.

[29] G. Dimarco, L. Pareschi, Numerical methods for kinetic equations, Acta Numerica, 32 (2014), pp. 369-520.

[30] G. Dimarco, L. Pareschi, Implicit-explicit linear multistep methods for stiff kinetic equations, SIAM J. Numer. Anal., 55 (2017), pp. 664-690.

[31] G. Dimarco, L. Pareschi, G. Samaey, Asymptotic Preserving Monte Carlo methods for transport equations in the diffusive limit, SIAM J. Sci. Comput., 40 (2018), pp. 504-528.

[32] F. Filbet, L. Pareschi, T. Rey, On steady-state preserving spectral methods for homogeneous Boltzmann equations, C. R. Math. Acad. Sci. Paris, 353 (2015), pp. 309-314. 
[33] F. Filbet, S. Jin, A class of asymptotic preserving schemes for kinetic equations and related problems with stiff sources, J. Comp. Phys., 229 (2010), 7625-7648.

[34] E. Hairer, C. Lubich, G. Wanner, Geometric Numerical Integration. StructurePreserving Algorithms for Ordinary Differential Equations, Springer, Berlin, (2002).

[35] T. Homolle, N. Hadjiconstantinou, A low-variance deviational simulation Monte Carlo for the Boltzmann equation, J. Comp. Phys., 226 (2007), pp. 2341-2358.

[36] T. Homolle, N. Hadjiconstantinou, Low-variance deviational simulation Monte Carlo, Phys. Fluids, 19 (2007), 041701.

[37] S. JIN, Efficient Asymptotic-Preserving (AP) schemes for some multiscale kinetic equations, SIAM J. Sci. Comput., 21 (1999), pp. 441-454.

[38] A. KLAR, An asymptotic-induced scheme for non stationary transport equations in the diffusive limit, SIAM J. Numer. Anal., 35 (1998), pp. 1073-1094.

[39] A. Klar, H. Neunzert, J. Struckmeier, Transition from kinetic theory to macroscopic fluid equations: a problem for domain decomposition and a source for new algorithms, Transport Theory and Statistical Physics, 29 (2000), pp. 93-106.

[40] K. Krycki, C. Berthon, M. Frank, R. Turpault, Asymptotic preserving numerical schemes for a nonclassical radiation transport model for atmospheric clouds, Math. Meth. Applied Sci., 36 (2013), pp. 2101-2116.

[41] P. Lafitte, G. Samaey, Asymptotic-preserving projective integration schemes for kinetic equations in the diffusion limit, SIAM J. Sci. Comp., 34 (2012), pp. 579-602.

[42] H. P. Le, B. Yan, R. E. Caflisch, J.-L. Cambier, Monte Carlo simulation of excitation and ionization collisions with complexity reduction, J. Comput. Phys., 346 (2017), pp. 480496.

[43] M. Lemou, Relaxed micro-macro schemes for kinetic equations, C. R. Math. Acad. Sci. Paris, 348 (2010), pp. 455-460.

[44] M. Lemou, L. Mieussens, A new asymptotic preserving scheme based on micro-macro formulation for linear kinetic equations in the diffusion limit, SIAM J. Sci. Comp., 31 (2008), pp. 334-368.

[45] Q. LI, J. Lu, W. Sun, Diffusion approximations and domain decomposition method of linear transport equations: Asymptotics and numerics, J. Comp. Phys., 292 (2015), pp. 141-167.

[46] Q. LI, J. Lu, W. Sun, Half-space Kinetic Equations with General Boundary Conditions, Math. Comp., 86 (2016), pp. 1269-1301.

[47] S. LiU, Monte Carlo strategies in scientific computing, Springer, 2004.

[48] E. Loevbak, G. Samaey, S. Vandewalle, A multilevel Monte Carlo method for asymptotic-preserving particle schemes, 2019, submitted. 
[49] C. Mouhot, L. Pareschi, Fast algorithms for computing the Boltzmann collision operator, Math. Comp., 75 (2006), pp. 1833-1852.

[50] K. NAnBu, Direct simulation scheme derived from the Boltzmann equation, J. Phys. Soc. Japan, 49 (1980), pp. 2042-2049.

[51] H. Neunzert, J. Struckmeier, Boltzmann simulation by particle methods, Proceeding "Boltzmannn's legacy 150 years after his birth".

[52] H. Neunzert, J. Struckmeier, Particle methods for the Boltzmann equation, Acta numerica, 4 (1995), pp. 417-457.

[53] L. Pareschi, G. Russo, An introduction to Monte Carlo methods for the Boltzmann equation, ESAIM Proc., 10 (1999), pp. 35-76.

[54] L. Pareschi, G. Toscani, Interacting Multiagent Systems: Kinetic Equations and Monte Carlo Methods, Oxford University Press, 2013.

[55] G. A. Radtke, J-P. M. Peraud, N. Hadjiconstantinou, On efficient simulations of multiscale kinetic transport, Phil. Trans. Royal Soc. A, 371 (2013), 20120182.

[56] W. Ren, H. Liu, S. Jin, An Asymptotic-Preserving Monte Carlo method for the Boltzmann equation, J. Comp. Phys., 276 (2014), pp. 380-404.

[57] G. Strang, On the construction and the comparison of difference schemes, SIAM J. Numer. Anal., 5 (1968), pp. 506-517.

[58] S. Tiwari, A. Klar, S. Hardt, A particle-particle hybrid method for kinetic and continuum equations, J. Comput. Phys., 228 (2009), pp. 7109-7124.

[59] B. YAN, A hybrid method with deviational particles for spatial inhomogeneous plasma, J. Comput. Phys., 309 (2016), pp. 18-36.

[60] B. Yan, R. CAflisch, A Monte Carlo method with negative particles for Coulomb collisions, J. Comput. Phys., 298 (2015), pp. 711-740. 